\title{
Genetic Diversity of Grapevine virus A in Washington and California Vineyards
}

\author{
Olufemi J. Alabi, Maher Al Rwahnih, Tefera A. Mekuria, and Rayapati A. Naidu
}

First, third, and fourth authors: Department of Plant Pathology, Washington State University, Irrigated Agriculture Research and Extension Center, Prosser 99350; and second author: Department of Plant Pathology, University of California, Davis 95616.

Current address of O. J. Alabi: Department of Plant Pathology \& Microbiology, Texas A\&M AgriLife Research \& Extension Center, Weslaco 78596.

Accepted for publication 17 October 2013.

\begin{abstract}
Alabi, O. J., Al Rwahnih, M., Mekuria, T. A., and Naidu, R. A. 2014. Genetic diversity of Grapevine virus $A$ in Washington and California vineyards. Phytopathology 104:548-560.

Grapevine virus A (GVA; genus Vitivirus, family Betaflexiviridae) has been implicated with the Kober stem grooving disorder of the rugose wood disease complex. In this study, 26 isolates of GVA recovered from wine grape (Vitis vinifera) cultivars from California and Washington were analyzed for their genetic diversity. An analysis of a portion of the RNAdependent RNA polymerase (RdRp) and complete coat protein (CP) sequences revealed intra- and inter-isolate sequence diversity. Our results indicated that both $\mathrm{RdRp}$ and $\mathrm{CP}$ are under strong negative selection based on the normalized values for the ratio of nonsynonymous substitutions per nonsynonymous site to synonymous substitutions per synony-

clustering. In contrast, the RdRp-based phylogenetic tree indicated segregation of GVA isolates from California and Washington into six clades, independent of geographic origin or cultivar. Phylogenetic network coupled with recombination analyses showed putative recombination events in both RdRp and CP sequence data sets, with more of these events located in the CP sequence. The preponderance of divergent variants of GVA co-replicating within individual grapevines could increase viral genotypic complexity with implications for phylogenetic analysis and evolutionary history of the virus. The knowledge of genetic diversity of GVA generated in this study will provide a foundation for elucidating the epidemiological characteristics of virus populations at different scales and implementing appropriate management strategies for minimizing the spread of genetic variants of the virus by vectors and via planting materials supplied to nurseries and grape growers.
\end{abstract} mous site. A global phylogenetic analysis of CP sequences revealed segregation of virus isolates into four major clades with no geographic
Additional keywords: selection pressure.
Grapevine virus A (GVA), the type member of the genus Vitivirus in the family Betaflexiviridae, is widespread in many grapevine-growing regions of the world (7). GVA, one of the extensively studied vitiviruses infecting grapevines (Vitis spp.), has been implicated with Kober stem grooving disorder of the rugose wood disease complex (39) and with Shiraz disease (SD) in South Africa and Australia (20). The virus is known to be transmitted in a semipersistent manner between grapevines by several species of mealybugs $(16,22,33,53,60)$ and soft scale insects $(11,25)$. The virus can also be transmitted mechanically by sap inoculation (4) and by mealybugs (46) to herbaceous hosts. The $\approx 800 \mathrm{~nm}$ long, slightly flexuous filamentous particles of GVA is composed of a positive-sense, single-stranded RNA with a genome size varying between 7,342 and 7,471 nucleotides (nt), excluding a poly-A tail at the $3^{\prime}$ terminus $(7,41)$. The genome encodes five open reading frames (ORFs 1-5; Fig. 1A) and these ORFs are expressed through at least two nested sets of subgenomic RNAs (13). Functions have been assigned for proteins encoded by ORF1 (194-kDa polyprotein involved in virus replication), ORF3 (31-kDa movement protein), ORF4 (21.5-kDa coat protein $[\mathrm{CP}])$, and ORF5 (10-kDa protein with putative nucleic acid binding property and RNA silencing suppressor activity) of the virus $(14,24,40,41,59)$. The $19-\mathrm{kDa}$ protein encoded by ORF2 of the virus was suggested to be involved in transmission by mealybugs (15).

Corresponding author: R. A. Naidu; E-mail address: naidu.rayapati@wsu.edu

http://dx.doi.org/10.1094/PHYTO-06-13-0179-R

(C) 2014 The American Phytopathological Society
Previous studies on GVA have shown considerable variability in the genome size, nucleotide identity (ranging between 70 and $100 \%$ ), and biological characteristics on certain indicator hosts $(7,42,43)$. Additional studies using variant-specific reverse transcription-polymerase chain reaction (RT-PCR), single-strand conformation polymorphism, RT-PCR-restriction fragment length polymorphism, and $\mathrm{CP}$ sequence analyses $(18,21,43,57)$ have indicated genetic diversity among GVA isolates. These studies have led to segregation of virus isolates into three molecular variants, designated as group I, II, and III. Further, variants of individual groups of GVA were shown to be present as mixed populations in a single grapevine $(18,21,43)$. Some of these molecular variants have also been shown to have distinct biological properties. For instance, grapevines showing symptoms of SD have been consistently found to harbor molecular variants belonging to group II in South Africa (18) and Australia (20), whereas isolates belonging to group III were generally not associated with SD (18). In addition, GVA isolates of each group showed differences in symptoms in manually inoculated Nicotiana benthamiana (21), suggesting the presence of highly heterogeneous and divergent strains of the virus with differing biological and virulence characteristics.

The above studies were conducted using the $3^{\prime}$-terminal sequences of GVA isolates largely derived from Europe, South Africa, and Australia. Moreover, only a limited number of virus isolates from American vineyards were included in these studies (20). To address this knowledge gap, we collected GVA isolates from California and Washington, two states accounting for nearly 95\% of the grape production in the United States, and analyzed the genetic diversity and population structure of the virus using 
two functional classes of virus genes, a transcription-related (RNA-dependent RNA polymerase [RdRp]) and a structural (CP) gene. The results showed presence of divergent sequence variants in both Washington and California vineyards and occurrence of distinct sequence variants as mixtures in individual grapevines. Through an analysis of genetic variability among two functional classes of genes and across virus subpopulations, the present study provided valuable information on genotypic complexity of GVA for advancing our understanding of virus epidemiology in vineyards. The results will help in designing reliable diagnostic tools for improving the sanitary status of planting materials provided to nurseries and grape growers.

\section{MATERIALS AND METHODS}

Plant samples. GVA isolates analyzed in this study were collected between 2005 and 2010 from different wine grape cultivars from commercial vineyards in the Yakima Valley of Washington State and grapevine collections maintained by the Foundation Plant Services (FPS), University of California, Davis, CA (17). Isolates from Washington were derived from own-rooted grapevines, whereas source grapevines for GVA isolates from California came originally from grafted vines prior to being planted as own-rooted vines at the FPS Experimental Field Station (1). A total of 26 virus isolates (16 from California and 10 from Washington) derived from 13 different red- and white-berried grapevine cultivars (Table 1) were evaluated in this study. An isolate represents virus sequences derived from a single grapevine.

Sample processing, nucleic acid extraction, and RT-PCR. Samples from Washington were prepared as described by Rowhani et al. (47) and one tube-single step RT-PCR assays were performed as described earlier (1). In the case of California samples, total RNA extracted from individual samples were subjected to a two-step RT-PCR as described earlier (1). For each isolate, approximately 740-base pair (bp) DNA fragment was amplified using primers GVA-CPF6356 (5'-GATACYCTAGT TATGCCAGA-3') and GVA-CPR7096 (5'-GCACCACACTTA CACACATTC- $3^{\prime}$ ) covering the full-length $\mathrm{CP}$ gene and flanking sequences (Fig. 1). Concurrently, a portion of the RdRp was amplified from each isolate using primers GVA-RdRp3987F (5'-ACMTCWGAYGAYACDGCHAC-3') and GVA-RdRp4894R (5'-CTCATYCKCCANCCRCAGAA-3') to yield approximately 900-bp DNA fragment (Fig. 1). These primers were designed based on consensus sequences of several isolates of GVA (Table 1). RT was carried out at $52^{\circ} \mathrm{C}$ for $1 \mathrm{~h}$ followed by 35 consecutive cycles of PCR (denaturation at $94^{\circ} \mathrm{C}$ for $30 \mathrm{~s}$, annealing at $55^{\circ} \mathrm{C}$ for $45 \mathrm{~s}$, and extension at $72^{\circ} \mathrm{C}$ for $30 \mathrm{~s}$ ) with a final extension for $7 \mathrm{~min}$ at $72^{\circ} \mathrm{C}$ in a GeneAmp PCR System 9700 (Applied Biosystems, CA). Aliquots of RT-PCR products were resolved on $1.2 \%$ agarose gels prestained with GelRed as described previously (1). A 1-kb Plus DNA ladder (Invitrogen Corp., Carlsbad, CA) was run in parallel in the same gel to verify size of amplicons specific to CP and RdRp.

Cloning, sequencing, and computational analyses. The $\mathrm{CP}$ specific DNA fragments derived from Washington samples were ligated directly into pCR2.1 vector (Invitrogen Corp.). Due to nonspecific amplifications, owing to the degeneracy of the primers, the right size RdRp-specific bands were first gel-eluted (QIAEX II Gel Extraction Kit; Qiagen Inc., Valencia, CA) prior to their ligation into pCR2.1 vector. Both CP- and RdRp-specific amplicons obtained from California samples were first gel-eluted prior to cloning into the pGEM-T Easy vector using kits and pro-

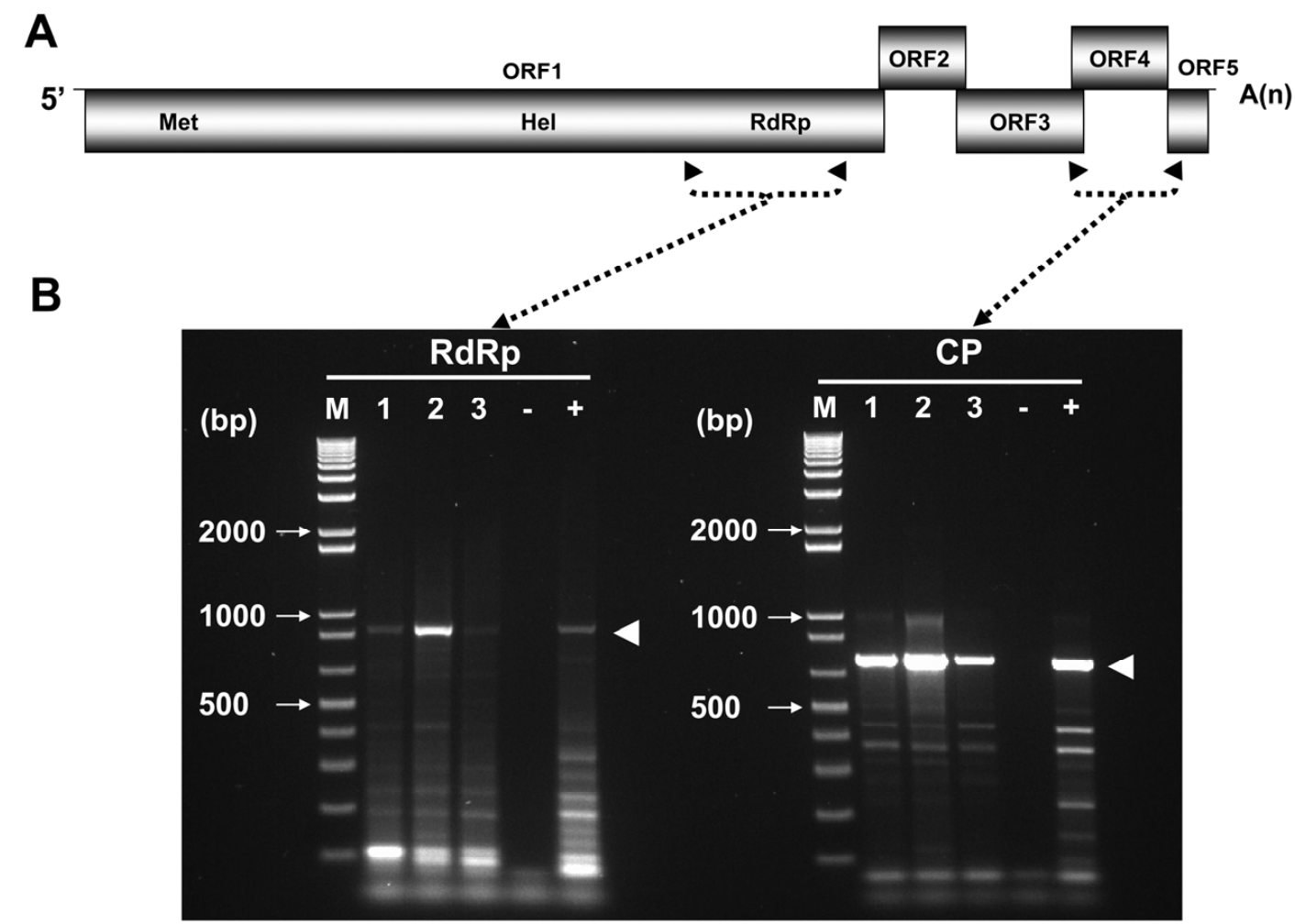

Fig. 1. A, Diagrammatic representation of the Grapevine virus A (GVA) genome showing the positions of different open reading frames (ORFs) as open boxes. The genome map is drawn based on the complete genome sequence of GVA isolate GTG11-1 from South Africa (DQ855084). ORF1, 194-kDa polyprotein containing motifs of replication-associated proteins (Met, methyl-transferase; Hel, helicase; RdRp, RNA-dependent RNA polymerase); ORF2, 19-kDa protein suggested to be involved in mealybug transmission; ORF3, movement protein; ORF4, coat protein (CP); ORF5, P10 RNA-binding protein (literature citation 7 provides details). B, Agarose gel electrophoresis of gene-specific RT-PCR products. Primers specific to a portion of the RdRp and primers encompassing the CP coding sequences were used to amplify $\approx 900$ and $\approx 740$ base pair (bp) DNA fragments (lanes 1 to 3 , indicated by white arrowhead), respectively, from each isolate. Both pairs of primers were designed based on consensus sequences of GVA isolates available in GenBank (Table 1). M, 1Kb Plus DNA Ladder (size of three fragments in base pairs [bp] is indicated by arrow on left); +, sample from GVA-positive grapevine; and -, sample from GVA-negative grapevine. 
cedures described earlier (1). The ligation products were transformed into chemically competent Escherichia coli cells according to the manufacturer's instructions (Invitrogen Corp). Selection of positive recombinant clones, plasmid DNA purification, and sequencing were done as described previously (1). Following analysis of haplotype diversity for the three initially sequenced independent clones per amplicon, additional clones were sequenced whenever distinct haplotypes were found in a single isolate. Clones sharing 98 to $100 \%$ nucleotide identity were considered as a single haplotype. Multiple sequence alignments were done using the partial order alignment (POA) tool (23) and the aligned sequences were edited visually and by using the ClustalW subsequence realignment tool (54) implemented in MEGA5 (52). The aligned sequences were used to estimate sequence identities as described previously (1).

Phylogenetic networks and recombination analyses. A quick scan for signatures of recombination was performed for each gene-specific sequence data set using the single breakpoint scanning (SBP) and genetic algorithm recombination detection (GARD) methods (32) and phylogenetic networks via the Neighbor-Net method (27). Both SBP and GARD methods were implemented by the Datamonkey software (31) while the Neighbor-Net method was implemented in SplitsTree v4.10 (27). Finally, aligned sequences specific to both the RdRp and CP were scanned, using seven recombination detection programs implemented in RDP v.3.44 software (36), for evidence of recombination and to determine acceptable putative recombination events, potential recombinants, and their parental sequences. Putative recombinant progenies were subsequently excluded from phylogenetic and population genetics analyses.

Phylogenetic analysis. RdRp- and $\mathrm{CP}$-specific sequences derived from this study, together with corresponding sequences extracted from GenBank (Table 1), were aligned and evolutionary relationships inferred using the maximum likelihood (ML) (44), neighbor joining (NJ) (50), and minimum evolution (ME) (48) methods with 1,000 bootstrap replications. Complete genome sequences of 12 GVA sequences available in GenBank (Table 1) were also used in phylogenetic analysis and the number and pattern of clustering of the isolates on the derived tree used for comparisons with the RdRp- and CP-derived trees.

Population genetic analysis and determination of selection pressure acting on RdRp and CP genes. Aligned sequences of

TABLE 1. List and identifiers of Grapevine virus A isolates analyzed in this study and global isolates of the virus

\begin{tabular}{|c|c|c|c|c|c|}
\hline Taxon ID & Source & Cultivar $^{\mathrm{a}}$ & Cultivar type & Genomic region $^{b}$ & GenBank accession number \\
\hline CBPR116 & California & Primitivo CL-4 & Red wine grape & $\mathrm{CP}$ & KF013763-766 \\
\hline CBSM119 & California & Sami-S1 & Red table grape & $\mathrm{RdRp}, \mathrm{CP}$ & KF013704-712, KF013767-770 \\
\hline H6TM2-3 & California & Tamar-S1 & White wine/table grape & $\mathrm{RdRp}, \mathrm{CP}$ & KF013713-720, KF013779-785 \\
\hline LREP100 & California & Emperor & Red wine/table grape & $\mathrm{RdRp}, \mathrm{CP}$ & KF013721-727, KF013810-811 \\
\hline LV94-02 & California & Unknown & Unknown & $\mathrm{CP}$ & KF013812-815 \\
\hline LVCH92-04 & California & Chardonnay & White wine grape & $\mathrm{RdRp}, \mathrm{CP}$ & KF031728-730, KF013816-818 \\
\hline LVCH92-07 & California & Chardonnay & White wine grape & $\mathrm{RdRp}, \mathrm{CP}$ & KF031738-742, KF013819-823 \\
\hline LVCH92-09 & California & Chardonnay & White wine grape & $\mathrm{CP}$ & KF031824-827 \\
\hline LVCH94-04 & California & Chardonnay & White wine grape & $\mathrm{CP}$ & KF031828-829 \\
\hline LVCS92-06 & California & Cabernet Sauvignon & Red wine grape & $\mathrm{RdRp}, \mathrm{CP}$ & KF031731-737, KF013830-835 \\
\hline LVMB92-10 & California & Malbec & Red wine grape & $\mathrm{CP}$ & KF031836-840 \\
\hline LVSB91-02 & California & Sauvignon Blanc & White wine grape & $\mathrm{CP}$ & KF031841 \\
\hline LVZT93-09 & California & Zante & $\ldots$ & $\mathrm{RdRp}, \mathrm{CP}$ & KF031743-754, KF013842 \\
\hline PACF94-142 & California & Cabernet Franc & Red wine grape & $\mathrm{CP}$ & KF031843-849 \\
\hline PLCF95-413 & California & Cabernet Franc & Red wine grape & $\mathrm{CP}$ & KF031850-854 \\
\hline HRPCH1 & Washington & Chardonnay & White wine grape & $\mathrm{RdRp}, \mathrm{CP}$ & KF031694, KF013797 \\
\hline HHCS1 & Washington & Cabernet Sauvignon & Red wine grape & $\mathrm{RdRp}, \mathrm{CP}$ & KF031685-686, KF013786-787 \\
\hline HHCS2 & Washington & Cabernet Sauvignon & Red wine grape & $\mathrm{RdRp}, \mathrm{CP}$ & KF031687, KF013788-789 \\
\hline HHCS3 & Washington & Cabernet Sauvignon & Red wine grape & RdRp, CP & KF031688-690, KF013790-792 \\
\hline CSOT1 & Washington & Cabernet Sauvignon & Red wine grape & $\mathrm{CP}$ & KF031771-774 \\
\hline CSOT2 & Washington & Cabernet Sauvignon & Red wine grape & $\mathrm{CP}$ & KF031775-778 \\
\hline HHPN1 & Washington & Pinot Noir & Red wine grape & $\mathrm{RdRp}, \mathrm{CP}$ & KF031691-693, KF013793-796 \\
\hline BVPN1 & Washington & Pinot Noir & Red wine grape & $\mathrm{RdRp}, \mathrm{CP}$ & KF031680-682, KF013755-757 \\
\hline BVPN2 & Washington & Pinot Noir & Red wine grape & $\mathrm{RdRp}, \mathrm{CP}$ & KF031683-684, KF013758-762 \\
\hline VHLM1 & Washington & Lemberger & Red wine grape & $\mathrm{RdRp}, \mathrm{CP}$ & KF031702-703, KF013806-809 \\
\hline SLWZF1 & Washington & Zinfandel & Red wine grape & $\mathrm{RdRp}, \mathrm{CP}$ & KF031695-701, KF013798-805 \\
\hline AF494187 & Brazil & Unknown & Unknown & $\mathrm{CP}$ & AF494187 \\
\hline MT25/7 & Czech Republic & Muller-Thurgau & White wine grape & $\mathrm{CP}$ & EU008561 \\
\hline MT43/25 & Czech Republic & Muller-Thurgau & White wine grape & $\mathrm{CP}$ & EU008560 \\
\hline Is 151 & Italy & NA & NA & Complete genome & X75433 \\
\hline $92 / 778$ & South Africa & Shiraz & Red wine grape & $\mathrm{CP}$ & AF441234 \\
\hline GTG11-1 & South Africa & Shiraz & Red wine grape & Complete genome & DQ855084 \\
\hline AY340581 & Brazil & NA & NA & $\mathrm{CP}$ & AY340581 \\
\hline MSH18-1 & South Africa & Shiraz & Red wine grape & $\mathrm{CP}$ & DQ855085 \\
\hline JP98 & South Africa & Shiraz & Red wine grape & $\mathrm{CP}$ & AF441235 \\
\hline GTG1-2 & South Africa & Shiraz & Red wine grape & Complete genome & DQ855086 \\
\hline P163-M5 & South Africa & Cinsaut Blanc & White wine grape & Complete genome & DQ855082 \\
\hline KWVMo4-1 & South Africa & Merlot & Red wine grape & Complete genome & DQ855083 \\
\hline GTG1SD-1 & South Africa & Shiraz & Red wine grape & Complete genome & DQ855081 \\
\hline BMo32-1 & South Africa & Merlot & Red wine grape & Complete genome & DQ855087 \\
\hline P163-1 & South Africa & Cinsaut Blanc & White wine grape & Complete genome & DQ855088 \\
\hline GTR1-1 & South Africa & Shiraz & Red wine grape & Complete genome & DQ787959 \\
\hline LQ58 & China & NA & NA & $\mathrm{CP}$ & DQ911145 \\
\hline PA3 & Israel & NA & NA & Complete genome & AF007415 \\
\hline AY244516 & Israel & NA & NA & Complete genome & AY244516 \\
\hline $3138-03$ & Canada & NA & NA & Complete genome & JX559641 \\
\hline
\end{tabular}

${ }^{a}$ Details of cultivars were obtained from http://ngr.ucdavis.edu/varietylist.cfm. NA = not available.

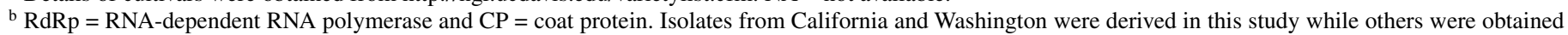
from the GenBank. 
CP- and RdRp-specific data set were used for determining population genetic parameters. These include, selection pressures, ratio of nonsynonymous substitutions per nonsynonymous site $(\mathrm{d} N)$ to synonymous substitutions per synonymous site $(\mathrm{d} S)$, average evolutionary divergence over sequence pairs, degree of genetic differentiation, presence of subdivisions among GVA populations, Tajima's D test of neutrality (51), and McDonald and Kreitman test of neutrality (37) to examine selection on proteins between GVA phylogroups. These analyses were performed as described previously $(1,2,29)$ for each sequence variant group using several software programs including Datamonkey software (31) (http://www.datamonkey.org/), MEGA5 (52), and DnaSP software version 5.10.01 (35).

\section{RESULTS}

RT-PCR amplification of RdRp- and CP-specific products. Using gene-specific primers, $\approx 900$ - and $\approx 740$-bp amplicons corresponding respectively to RdRp and CP (Fig. 1), were amplified from a total of 26 isolates. As shown in Table 1, viral genomic segments were successfully amplified from each of the 26 isolates. However, amplicons specific to the RdRp were obtained from a total of $16 \mathrm{GVA}$ isolates, whereas $\mathrm{CP}$-specific amplicons were derived for all 26 isolates.

Phylogenetic network and recombination analyses. Since potential occurrence of recombination may produce different alignments with contrasting evolutionary histories (45), we scanned the RdRp and CP sequence haplotypes for signatures of recombination and presence of putative recombination events. A phylogenetic network analysis performed for each of the sequence data sets using the Neighbor-Net method implemented by the SplitsTree4 software (27) resulted in non-tree-like phylogenetic networks among sequences of both the RdRp- and CPspecific data sets (Fig. 2). This suggests that recombination events could be contributing to the evolution of GVA. Further evidence for fingerprints of recombination in both genomic regions was found by using the SBP and GARD recombination detection methods (32). Subsequent scans using the RDP3-implemented programs led to the detection of several putative recombination events, chimera sequences and potential parental sequences (Table 2). Determination of authentic events was based on threshold levels described previously (1), whereby only an event detected by at least four of the seven RDP-implemented programs is considered acceptable. Based on these criteria, six putative recombination events were identified when global RdRp gene sequences (75 obtained from this study [accession nos. KF013680-754] and 11 extracted from GenBank [Table 1]) were analyzed for recombination. The RdRp-specific events involved sequence variants of four isolates from California (LVCS92-06, LVZT93-09, H6TM2-3, and LREP100) as putative recombinants and parentals, along with an isolate from South Africa (GTR1-1), as potential major and minor parental sequences (Table 2). Five of the six putative recombination events in the $\mathrm{RdRp}$ constitute intra-isolate recombination events, wherein divergent parental sequences exchanged genetic materials leading to the birth of a chimera sequence and the majority of them (four out of six) involved parentals belonging to phylogroup III (Table 2). Eight putative recombination events were detected when global CP gene-specific sequences (100 obtained from this study [accession nos. KF013755-854] and 20 extracted from GenBank [Table 1]) were analyzed for recombination. These events involved five isolates from California (LVCS92-06, CBSM119, LVMB92-10, H6TM2-3, and LVCH9209), five from Washington (BVPN2, CSOT1, CSOT2, HHPN1, and SLWZF1), and an isolate (AF494187) from Brazil as putative recombinants and other sequence variants of the same isolates as potential major and minor parental sequences (Table 2). Unlike recombinant events identified in the RdRp, only a few of the putative recombination events in the $\mathrm{CP}$ involved parentals and their recombinants resident in a single grapevine. One of the eight putative recombination events of the $\mathrm{CP}$ involved parental sequences belonging to phylogroup I, one event involved parental sequences belonging to phylogroup III, three events involved parentals belonging to phylogroups I and III, two involved parentals belonging to phylogroups II and III, and an additional one event involved parentals belonging to phylogroup II and a recombinant sequence. These results indicated that virus sequences belonging to phylogroup III have greater propensity for recombination than those belonging to other phylogroups. The putative recombinant progenies (Table 2) were excluded from subsequent phylogenetic and population genetic analyses described below.

Nucleotide diversity among RdRp- and CP-derived sequences. Using nonrecombinant sequence haplotypes, we calculated genetic distances for each gene-specific data set taking into account the appropriate models of best fit obtained using MEGA5 software (52). The overall mean value of nucleotide diversity was $0.209 \pm 0.007$ for the $\operatorname{RdRp}(80$ sequences: California $=45$, Washington $=24$, and GenBank $=11)$ and $0.168 \pm 0.010$ for the CP $(104$ sequences: California $=50$, Washington $=35$, and GenBank = 19). Thus, the mean value of nucleotide diversity is lower for the $\mathrm{CP}$ in comparison to the RdRp. Interestingly, the mean value of nucleotide diversity obtained using complete genome sequences of 12 GenBank isolates gave a value $(0.226 \pm$ 0.003 ) that was similar to the value obtained for $\mathrm{RdRp}$ sequences analyzed in this study.

Phylogenetic analysis of GVA isolates. We constructed the phylogeny of GVA isolates using RdRp- and CP-derived nucleotide sequences of haplotypes obtained from this study and corresponding sequences of isolates available in public databases (Table 1). All putative recombinant sequences identified by the RDP3-implemented programs (Table 2; Fig. 2) were excluded from these analyses. The evolutionary model that best fitted alignments of each gene-specific data set was determined as described previously (1). Subsequently, maximum likelihood (ML), minimum evolution (ME), and neighbor-joining (NJ) phylogenetic trees were constructed using the model that best fitted each sequence data set. Only the ME trees are shown since similar trees were inferred using all three methods (Fig. 3).

Global CP-specific sequences of GVA $($ Total $=105$; California $=$ 50, Washington $=35$, and GenBank $=20$ ) segregated into four major phylogroups with strong $(>70 \%)$ bootstrap support values (Fig. 3A). Based on the relative placements of previously analyzed reference sequences of GVA (18-20) (Table 1), the phylogroups were designated as groups I, II, III, and IV to maintain consistency in naming phylogroups. Group I comprised of sequences from California, Washington, Czech Republic, Italy, South Africa, Brazil, and Israel; group II included sequences from California, Washington, and South Africa; group III included sequences from California, Washington, South Africa, China, and Canada; and group IV comprised sequences from California, Washington, and Israel. These results indicated that sequences from California and Washington segregated into all four phylogroups (Fig. 3A) and showed no discernible pattern of geographical structuring. Further analysis showed that majority (36.5\%) of the $\mathrm{CP}$ sequences belonged to group III, followed by group I (33.7\%), group II (19.2\%), and group IV (10.6\%). The average evolutionary divergence over sequence pairs within each of the four CP-derived phylogroups ranged from $0.060 \pm 0.006$ to $0.102 \pm 0.008$ (Table 3 ) and those between groups ranged from $0.136 \pm 0.011$ to $0.228 \pm 0.015$ (Table 4 ).

Unlike the CP-based phylogram, 81 of the RdRp sequences $($ California $=45$, Washington $=24$, and GenBank $=12)$ segregated into six phylogroups with good $(\geq 57 \%)$ bootstrap support values (Fig. 3B). We identified four phylogroups (I to IV) based on the relative positions of the same reference isolates in the $\mathrm{CP}$ derived tree. In addition, we found two more clusters with very strong $(>90 \%)$ bootstrap supports and these were designated as 

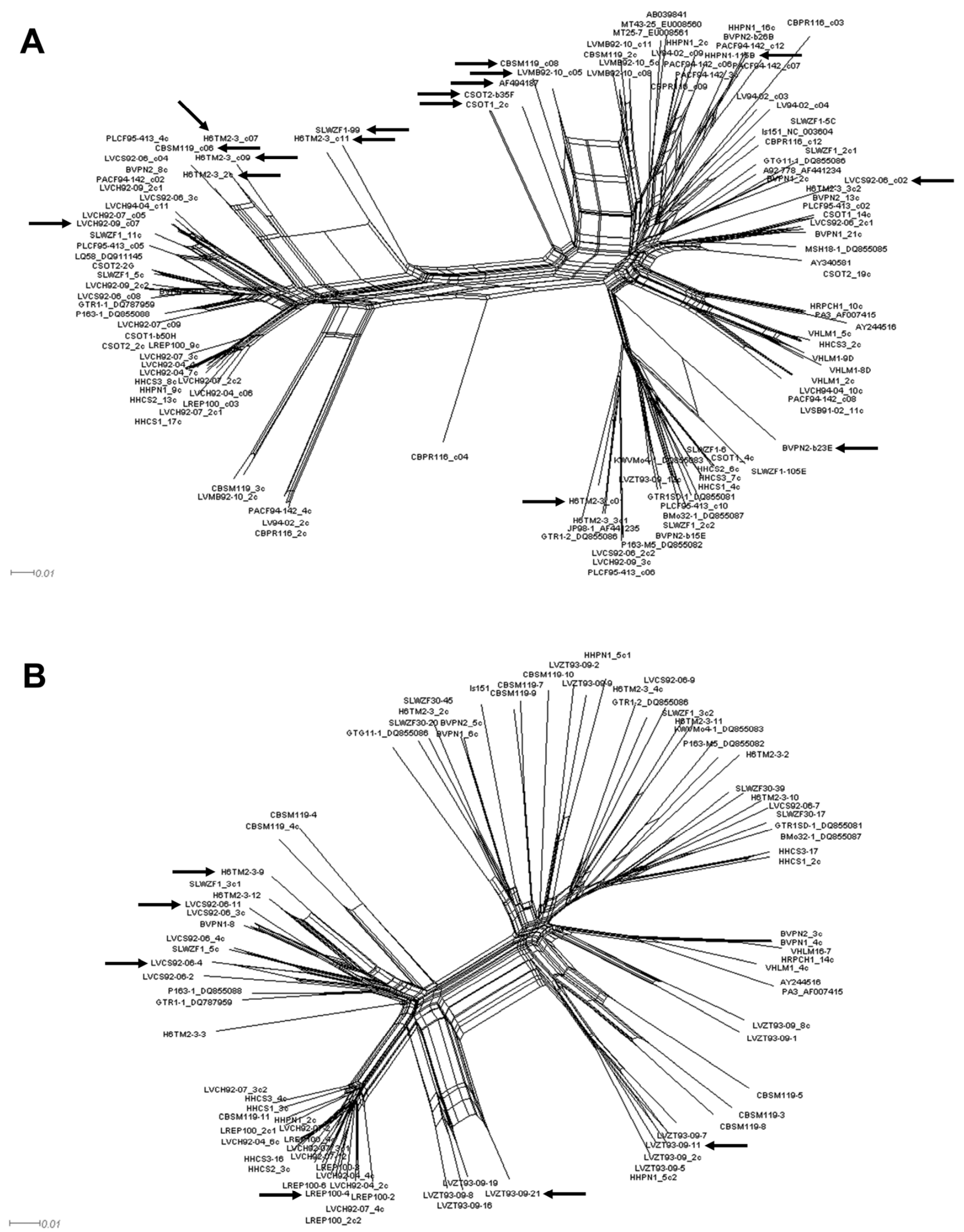

Fig. 2. Phylogenetic network trees depicting complex evolutionary scenarios among A, coat protein and B, RNA-dependent RNA polymerase sequences of Grapevine virus A. Putative recombinant sequences (indicated by black arrows) were identified by at least four of the seven RDP-implemented programs. These recombinants were excluded from phylogenetic trees presented in Figure 3. 
phylogroups V and VI. Group I comprised of sequences from California, Washington, Italy, and South Africa; group II included sequences from California, Washington, and South Africa; group III comprised of sequences from California, Washington, Canada, and South Africa; group IV included sequences from California, Washington, and Israel; and both groups V and VI included only sequences from California and Washington. Thus, RdRp sequences from both California and Washington were segregated into all six phylogroups (Fig. 3B). Further analysis showed that a majority $(43.8 \%)$ of the RdRp sequences belonged to group III, followed by group II (20\%), groups I and IV (11.3\% each), group V (8.8\%), and group VI (1.3\%). The average evolutionary divergence over sequence pairs within each of the six RdRp-derived phylogroups ranged from $0.121 \pm 0.006$ to $0.167 \pm 0.008$ (Table 3) and those between groups ranged from $0.203 \pm 0.009$ to $0.254 \pm 0.011$ (Table 4).

We used complete genome sequences of 12 GVA isolates available in GenBank (Table 1) for additional phylogenetic analy- sis. The results showed segregation of complete genome sequences into four distinct phylogroups (Fig. 3C), analogous to the CP-based phylogram (Fig. 3A). The existence of four groups was further confirmed by strong ( $>90 \%)$ bootstrap support at their nodes (Fig. 3C). Group I consisted of isolates from South Africa and Italy, group II consisted of isolates from South Africa, group III consisted of isolates from South Africa and Canada, and group IV consisted of isolates from Israel. Notably, the pattern of clustering of complete genome sequences of GVA isolates was in alignment with CP-derived trees, indicating that the evolutionary history of global populations of GVA can be inferred reliably using the CP coding sequences than RdRp sequences.

Haplotype diversities in individual grapevines. Initially three independent clones were sequenced per CP- and RdRpspecific amplicon obtained from a single grapevine and the level of their nucleotide sequence identities determined. Using a threshold of 98 to $100 \%$ nt identities among sequence haplotypes (1), additional clones were sequenced whenever distinct haplo-

TABLE 2. Putative intragenic recombination events in Grapevine virus A sequences ${ }^{\mathrm{a}}$

\begin{tabular}{|c|c|c|c|c|c|c|c|c|c|c|c|c|}
\hline \multirow[b]{2}{*}{ Event $^{\mathrm{b}}$} & \multicolumn{2}{|c|}{ Breakpoint } & \multirow[b]{2}{*}{$\operatorname{Recom}(\text { GenBank })^{c}$} & \multicolumn{2}{|c|}{ Parentals $^{\mathrm{d}}$} & \multicolumn{7}{|c|}{ Method (Average $P$ value) } \\
\hline & Start & End & & Major & Minor & $\mathrm{R}$ & $\mathrm{G}$ & B & M & $\mathrm{C}$ & $\mathrm{S}$ & $3 \mathrm{Seq}$ \\
\hline \multicolumn{13}{|l|}{$\mathrm{RdRp}$} \\
\hline 1 & $\mathrm{U}(3)$ & 354 & $\begin{array}{c}\text { LVCS92-06-4 } \\
(\text { KF013734) }\end{array}$ & $\begin{array}{r}\text { LVCS92-06-2 } \\
(98.7 \%)[\mathrm{III}]\end{array}$ & $\begin{array}{c}\text { LVCS92-06_4c } \\
(99.1 \%)[\mathrm{III}]\end{array}$ & - & $\begin{array}{l}1.49 \times \\
10^{-05}\end{array}$ & $\begin{array}{c}7.90 \times \\
10^{-10}\end{array}$ & $\begin{array}{c}1.19 \times \\
10^{-11}\end{array}$ & $\begin{array}{l}5.78 \times \\
10^{-12}\end{array}$ & $\begin{array}{l}1.05 \times \\
10^{-14}\end{array}$ & $\begin{array}{c}3.31 \times \\
10^{-20}\end{array}$ \\
\hline 2 & $\mathrm{U}(3)$ & 602 & $\begin{array}{l}\text { LVZT93-09-21 } \\
\text { (KF013754) }\end{array}$ & $\begin{array}{l}\text { LVZT93-09_2c } \\
(98.7 \%)[\mathrm{V}]\end{array}$ & $\begin{array}{l}\text { LVZT93-09-19 } \\
(97.7 \%)[\mathrm{III}]\end{array}$ & $\begin{array}{l}5.90 \times \\
10^{-03}\end{array}$ & - & $\begin{array}{l}4.02 \times \\
10^{-02}\end{array}$ & $\begin{array}{l}4.35 \times \\
10^{-05}\end{array}$ & $\begin{array}{l}3.02 \times \\
10^{-05}\end{array}$ & $\begin{array}{l}7.62 \times \\
10^{-28}\end{array}$ & $\begin{array}{c}1.43 \times \\
10^{-19}\end{array}$ \\
\hline 3 & $\mathrm{U}(3)$ & 646 & $\begin{array}{l}\text { H6TM2-3-9 } \\
\text { (KF013717) }\end{array}$ & U(GTR1-1) [III] & $\begin{array}{l}\text { H6TM2-3-12 } \\
(99.7 \%)[\mathrm{III}]\end{array}$ & - & - & - & $\begin{array}{l}2.08 \times \\
10^{-03}\end{array}$ & $\begin{array}{l}4.81 \times \\
10^{-03}\end{array}$ & $\begin{array}{l}8.77 \times \\
10^{-19}\end{array}$ & $\begin{array}{c}2.55 \times \\
10^{-17}\end{array}$ \\
\hline 4 & $\mathrm{U}(1)$ & $\mathrm{U}(417)$ & $\begin{array}{l}\text { LVCS92-06-11 } \\
\text { (KF013737) }\end{array}$ & $\begin{array}{l}\text { LVCS92-06_4c } \\
(100 \%)[\mathrm{III}]\end{array}$ & $\begin{array}{c}\text { LVCS92-06_3c } \\
(98.6 \%)[\mathrm{III}]\end{array}$ & - & $\begin{array}{l}4.40 \times \\
10^{-02}\end{array}$ & $\begin{array}{l}2.39 \times \\
10^{-02}\end{array}$ & $\begin{array}{l}3.70 \times \\
10^{-07}\end{array}$ & $\begin{array}{l}4.13 \times \\
10^{-07}\end{array}$ & $\begin{array}{l}1.77 \times \\
10^{-12}\end{array}$ & $\begin{array}{l}3.72 \times \\
10^{-15}\end{array}$ \\
\hline 5 & $\mathrm{U}(4)$ & 406 & $\begin{array}{l}\text { LVZT93-09-11 } \\
(\text { KF013751) }\end{array}$ & $\begin{array}{c}\text { LVZT93-09-7 } \\
(99.7 \%)[\mathrm{V}]\end{array}$ & $\begin{array}{l}\text { LVZT93-09_2c } \\
(99 \%)[\mathrm{V}]\end{array}$ & - & - & $\begin{array}{l}4.82 \times \\
10^{-03}\end{array}$ & $\begin{array}{l}1.39 \times \\
10^{-06}\end{array}$ & $\begin{array}{l}1.35 \times \\
10^{-06}\end{array}$ & $\begin{array}{l}1.11 \times \\
10^{-12}\end{array}$ & $\begin{array}{l}1.48 \times \\
10^{-13}\end{array}$ \\
\hline 7 & 341 & $\mathrm{U}(744)$ & $\begin{array}{l}\text { LREP100-4 } \\
\text { (KF013726) }\end{array}$ & $\begin{array}{l}\text { LREP100-3 } \\
(97.7 \%)[\mathrm{III}]\end{array}$ & $\begin{array}{l}\text { LREP100_4c (99\%) } \\
\text { [III] }\end{array}$ & - & - & - & $\begin{array}{l}2.76 \times \\
10^{-04}\end{array}$ & - & $\begin{array}{l}3.71 \times \\
10^{-05}\end{array}$ & $\begin{array}{c}1.45 \times \\
10^{-03}\end{array}$ \\
\hline \multicolumn{13}{|l|}{$\mathrm{CP}$} \\
\hline 1 & $\mathrm{U}(181)$ & $\mathrm{U}(597)$ & $\begin{array}{c}\text { BVPN2-b23E } \\
(\text { KF013761) }\end{array}$ & $\begin{array}{l}\text { CSOT2_2c (100\%) } \\
\text { [III] }\end{array}$ & $\begin{array}{l}\text { SLWZF1-105E } \\
(100 \%)[\mathrm{II}]\end{array}$ & - & $\begin{array}{l}7.67 \times \\
10^{-05}\end{array}$ & $\begin{array}{l}7.32 \times \\
10^{-11}\end{array}$ & $\begin{array}{l}1.50 \times \\
10^{-05}\end{array}$ & $\begin{array}{l}6.18 \times \\
10^{-09}\end{array}$ & - & $\begin{array}{l}1.60 \times \\
10^{-15}\end{array}$ \\
\hline 1 & $\mathrm{U}(184)$ & $\mathrm{U}(597)$ & $\begin{array}{l}\text { LVCS92-06_c02 } \\
\text { (KF013833) }\end{array}$ & $\begin{array}{l}\text { CSOT2_2c }(93.4 \%) \\
\text { [III] }\end{array}$ & $\begin{array}{l}\text { SLWZF1-105E } \\
(85.5 \%)[\mathrm{II}]\end{array}$ & - & $\begin{array}{l}7.67 \times \\
10^{-05}\end{array}$ & $\begin{array}{l}7.32 \times \\
10^{-11}\end{array}$ & $\begin{array}{l}1.50 \times \\
10^{-05}\end{array}$ & $\begin{array}{l}6.18 \times \\
10^{-09}\end{array}$ & - & $\begin{array}{l}1.60 \times \\
10^{-15}\end{array}$ \\
\hline 2 & $\mathrm{U}(17)$ & 383 & $\begin{array}{l}\text { CBSM119_c08 } \\
\text { (KF013770) }\end{array}$ & $\begin{array}{l}\text { LVMB92-10_2c } \\
(97 \%)[\mathrm{III}]\end{array}$ & $\begin{array}{l}\text { LVMB92-10_c08 } \\
(91 \%)[\mathrm{I}]\end{array}$ & - & - & $\begin{array}{l}1.89 \times \\
10^{-04}\end{array}$ & $\begin{array}{l}3.22 \times \\
10^{-04}\end{array}$ & $\begin{array}{l}6.74 \times \\
10^{-05}\end{array}$ & $\begin{array}{l}1.33 \times \\
10^{-16}\end{array}$ & $\begin{array}{c}4.64 \times \\
10^{-12}\end{array}$ \\
\hline 2 & $\mathrm{U}(1)$ & 383 & $\begin{array}{l}\text { LVMB92-10_c05 } \\
\text { (KF013838) }\end{array}$ & $\begin{array}{l}\text { LVMB92-10_2c } \\
(99.5 \%)[\mathrm{III}]\end{array}$ & $\begin{array}{l}\text { LVMB92-10_c08 } \\
(99.2 \%)[\mathrm{I}]\end{array}$ & - & - & $\begin{array}{l}1.89 \times \\
10^{-04}\end{array}$ & $\begin{array}{l}3.22 \times \\
10^{-04}\end{array}$ & $\begin{array}{l}6.74 \times \\
10^{-05}\end{array}$ & $\begin{array}{l}1.33 \times \\
10^{-16}\end{array}$ & $\begin{array}{c}4.64 \times \\
10^{-12}\end{array}$ \\
\hline 2 & $\mathrm{U}(17)$ & 465 & AF494187 & $\begin{array}{c}\text { LVMB92-10_2c } \\
(87.2 \%)[\mathrm{III}]\end{array}$ & $\begin{array}{l}\text { LVMB92-10_c08 } \\
(89.3 \%)[\mathrm{I}]\end{array}$ & - & - & $\begin{array}{l}1.89 \times \\
10^{-04}\end{array}$ & $\begin{array}{l}3.22 \times \\
10^{-04}\end{array}$ & $\begin{array}{l}6.74 \times \\
10^{-05}\end{array}$ & $\begin{array}{l}1.33 \times \\
10^{-16}\end{array}$ & $\begin{array}{c}4.64 \times \\
10^{-12}\end{array}$ \\
\hline 3 & 440 & $\mathrm{U}(597)$ & $\begin{array}{l}\text { CSOT1_2c } \\
(\text { KF013773) }\end{array}$ & $\begin{array}{l}\text { BVPN1_2c }(96.4 \%) \\
{[\mathrm{I}]}\end{array}$ & $\begin{array}{c}\text { SLWZF1_5C } \\
(100 \%)[\mathrm{I}]\end{array}$ & - & $\begin{array}{l}1.46 \times \\
10^{-06}\end{array}$ & $\begin{array}{l}8.24 \times \\
10^{-09}\end{array}$ & - & - & $\begin{array}{l}1.39 \times \\
10^{-06}\end{array}$ & $\begin{array}{l}1.02 \times \\
10^{-14}\end{array}$ \\
\hline 3 & 440 & $\mathrm{U}(597)$ & $\begin{array}{r}\text { CSOT2-b35F } \\
(\text { KF013778) }\end{array}$ & $\begin{array}{l}\text { BVPN1_2c }(96.4 \%) \\
{[\mathrm{I}]}\end{array}$ & $\begin{array}{c}\text { SLWZF1_5C } \\
(100 \%)[\mathrm{I}]\end{array}$ & - & $\begin{array}{l}1.46 \times \\
10^{-06}\end{array}$ & $\begin{array}{l}8.24 \times \\
10^{-09}\end{array}$ & - & - & $\begin{array}{l}1.39 \times \\
10^{-06}\end{array}$ & $\begin{array}{l}1.02 \times \\
10^{-14}\end{array}$ \\
\hline 4 & $\mathrm{U}(12)$ & 432 & $\begin{array}{c}\text { HHPN1-115B } \\
(\text { KF013796) }\end{array}$ & $\begin{array}{l}\text { HHPN1_2c } \\
(98.9 \%)[\mathrm{I}]\end{array}$ & $\begin{array}{c}\text { HHPN1_16c } \\
(100 \%)[\mathrm{I}]\end{array}$ & - & - & $\begin{array}{l}2.94 \times \\
10^{-03}\end{array}$ & $\begin{array}{l}3.25 \times \\
10^{-04}\end{array}$ & $\begin{array}{l}6.87 \times \\
10^{-04}\end{array}$ & - & $\begin{array}{c}9.81 \times \\
10^{-12}\end{array}$ \\
\hline 5 & 515 & $\mathrm{U}(596)$ & $\begin{array}{l}\text { H6TM2-3_c01 } \\
\text { (KF013782) }\end{array}$ & $\begin{array}{c}\text { H6TM2-3_3c1 } \\
(97.3 \%)[\mathrm{II}]\end{array}$ & $\begin{array}{c}\text { CBSM119_c06 } \\
(100 \%)[\mathrm{R}]\end{array}$ & $\begin{array}{l}4.15 \times \\
10^{-07}\end{array}$ & $\begin{array}{l}1.08 \times \\
10^{-06}\end{array}$ & - & $\begin{array}{c}1.81 \times \\
10^{-03}\end{array}$ & $\begin{array}{l}4.14 \times \\
10^{-03}\end{array}$ & - & $\begin{array}{c}4.42 \times \\
10^{-06}\end{array}$ \\
\hline 7 & 243 & $\mathrm{U}(456)$ & $\begin{array}{l}\text { LVCH92-09_c07 } \\
\text { (KF013827) }\end{array}$ & $\begin{array}{l}\text { LVCH92-09_2c1 } \\
(100 \%)[\mathrm{III}]\end{array}$ & $\begin{array}{l}\text { LVCH92-09_2c2 } \\
(100 \%)[\mathrm{III}]\end{array}$ & - & - & $\begin{array}{l}4.45 \times \\
10^{-02}\end{array}$ & $\begin{array}{l}5.40 \times \\
10^{-05}\end{array}$ & $\begin{array}{l}3.68 \times \\
10^{-04}\end{array}$ & $\begin{array}{l}3.10 \times \\
10^{-06}\end{array}$ & $\begin{array}{l}1.08 \times \\
10^{-06}\end{array}$ \\
\hline 15 & 75 & $\mathrm{U}(592)$ & $\begin{array}{l}\text { H6TM2-3_c11 } \\
(\text { KF013785) }\end{array}$ & $\begin{array}{c}\text { SLWZF1_2c1 } \\
(82.3 \%)[\mathrm{I}]\end{array}$ & $\begin{array}{l}\text { CSOT2_2c }(89.2 \%) \\
\text { [III] }\end{array}$ & - & $\begin{array}{l}1.03 \times \\
10^{-03}\end{array}$ & $\begin{array}{l}7.64 \times \\
10^{-08}\end{array}$ & $\begin{array}{l}5.19 \times \\
10^{-08}\end{array}$ & $\begin{array}{l}4.03 \times \\
10^{-08}\end{array}$ & $\begin{array}{l}9.41 \times \\
10^{-16}\end{array}$ & $\begin{array}{c}2.72 \times \\
10^{-12}\end{array}$ \\
\hline 15 & 129 & $\mathrm{U}(597)$ & $\begin{array}{l}\text { SLWZF1-99 } \\
\text { (KF013805) }\end{array}$ & $\begin{array}{c}\text { SLWZF1_2c1 } \\
(100 \%)[\mathrm{I}]\end{array}$ & $\begin{array}{l}\text { CSOT2_2c }(99.6 \%) \\
\text { [III] }\end{array}$ & - & $\begin{array}{l}1.03 \times \\
10^{-03}\end{array}$ & $\begin{array}{l}7.64 \times \\
10^{-08}\end{array}$ & $\begin{array}{c}5.19 \times \\
10^{-08}\end{array}$ & $\begin{array}{l}4.03 \times \\
10^{-08}\end{array}$ & $\begin{array}{l}9.41 \times \\
10^{-16}\end{array}$ & $\begin{array}{c}2.72 \times \\
10^{-12}\end{array}$ \\
\hline 16 & $\mathrm{U}(31)$ & $\mathrm{U}(593)$ & $\begin{array}{l}\text { CBSM119_c06 } \\
\text { (KF013769) }\end{array}$ & $\begin{array}{l}\text { H6TM2-3_3c1 } \\
(75 \%)[\mathrm{II}]\end{array}$ & $\begin{array}{c}\text { LVCH92-04_c06 } \\
(90.6 \%)[\mathrm{III}]\end{array}$ & - & - & - & $\begin{array}{l}1.31 \times \\
10^{-03}\end{array}$ & $\begin{array}{l}1.56 \times \\
10^{-03}\end{array}$ & $\begin{array}{c}9.91 \times \\
10^{-13}\end{array}$ & $\begin{array}{c}3.67 \times \\
10^{-07}\end{array}$ \\
\hline 16 & $\mathrm{U}(17)$ & $\mathrm{U}(593)$ & $\begin{array}{r}\text { H6TM2-3_2c } \\
(\text { KF013779) }\end{array}$ & $\begin{array}{c}\text { H6TM2-3_3c1 } \\
(76.2 \%)[\mathrm{II}]\end{array}$ & $\begin{array}{l}\text { LVCH92-04_c06 } \\
(91 \%)[\mathrm{III}]\end{array}$ & - & - & - & $\begin{array}{l}1.31 \times \\
10^{-03}\end{array}$ & $\begin{array}{l}1.56 \times \\
10^{-03}\end{array}$ & $\begin{array}{l}9.91 \times \\
10^{-13}\end{array}$ & $\begin{array}{c}3.67 \times \\
10^{-07}\end{array}$ \\
\hline 16 & $\mathrm{U}(3)$ & $\mathrm{U}(479)$ & $\begin{array}{l}\text { H6TM2-3_c07 } \\
\text { (KF013783) }\end{array}$ & $\begin{array}{l}\text { H6TM2-3_3c1 } \\
(100 \%)[\mathrm{II}]\end{array}$ & $\begin{array}{l}\text { LVCH92-04_c06 } \\
(92.5 \%)[\mathrm{III}]\end{array}$ & - & - & - & $\begin{array}{l}1.31 \times \\
10^{-03}\end{array}$ & $\begin{array}{l}1.56 \times \\
10^{-03}\end{array}$ & $\begin{array}{l}9.91 \times \\
10^{-13}\end{array}$ & $\begin{array}{l}3.67 \times \\
10^{-07}\end{array}$ \\
\hline 16 & $\mathrm{U}(17)$ & $\mathrm{U}(597)$ & $\begin{array}{l}\text { H6TM2-3_c09 } \\
\text { (KF013784) }\end{array}$ & $\begin{array}{c}\text { H6TM2-3_3c1 } \\
(76.5 \%)[\mathrm{II}]\end{array}$ & $\begin{array}{l}\text { LVCH92-04_c06 } \\
(89.1 \%)[\mathrm{III}]\end{array}$ & - & - & - & $\begin{array}{l}1.31 \times \\
10^{-03}\end{array}$ & $\begin{array}{l}1.56 \times \\
10^{-03}\end{array}$ & $\begin{array}{l}9.91 \times \\
10^{-13}\end{array}$ & $\begin{array}{l}3.67 \times \\
10^{-07}\end{array}$ \\
\hline
\end{tabular}

${ }^{a}$ Only events supported by at least four of the different RDP3-implemented methods (36) are reported. The support probability for each method is shown.

Nucleotide numbering of breakpoints corresponds to the aligned sequences.

${ }^{\mathrm{b}} \mathrm{RdRp}=\mathrm{RNA}$-dependent RNA polymerase and $\mathrm{CP}=$ coat protein .

${ }^{c}$ Recombinant (GenBank accession number).

$\mathrm{d}$ The phylogenetic group to which each parental sequence belonged, based on the phylogenetic trees (Fig. 3A and B), is shown in square brackets. 
types were found among the three initially sequenced clones. We then determined the number and diversity of haplotypes obtained within individual grapevines. The results showed considerable haplotype diversity in individual isolates based on RdRp- and CPspecific sequences (Table 5). There was considerable haplotype diversity among sequences derived from each isolate analyzed in this study with the exception of two (HRPCH1 and HHCS2) and three (HRPCH1, LVSB91-02, and LVZT93-09) isolates for sequences derived from RdRp and CP, respectively (Table 5). The number of distinct haplotypes specific to RdRp present in
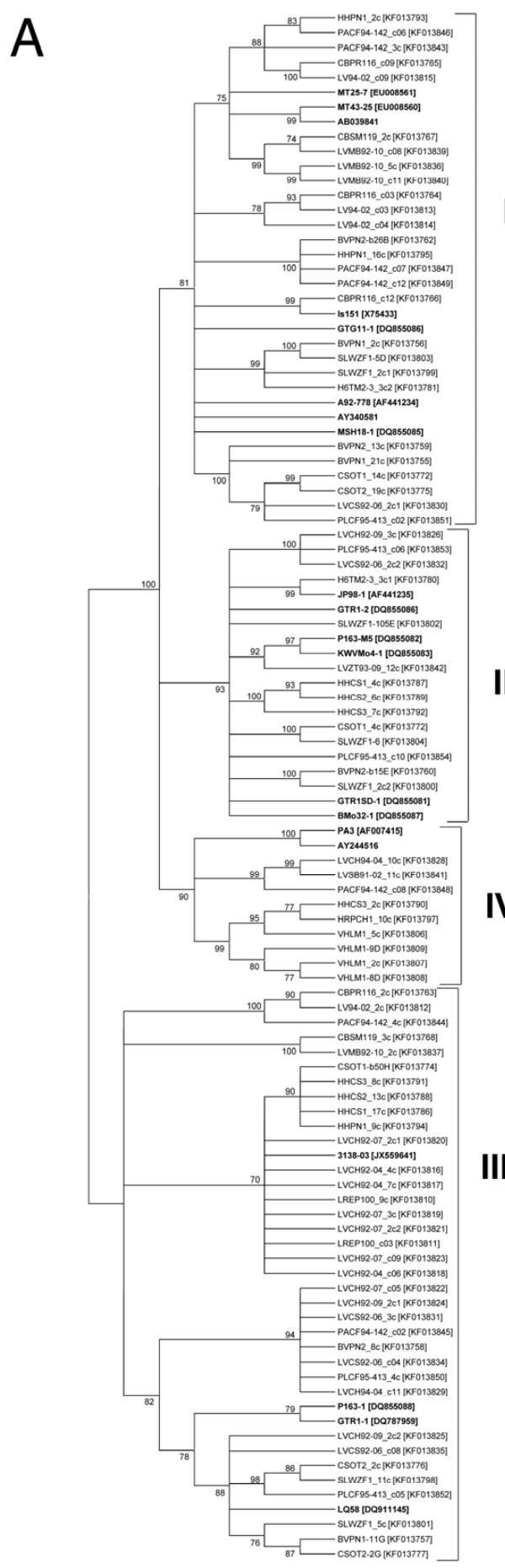

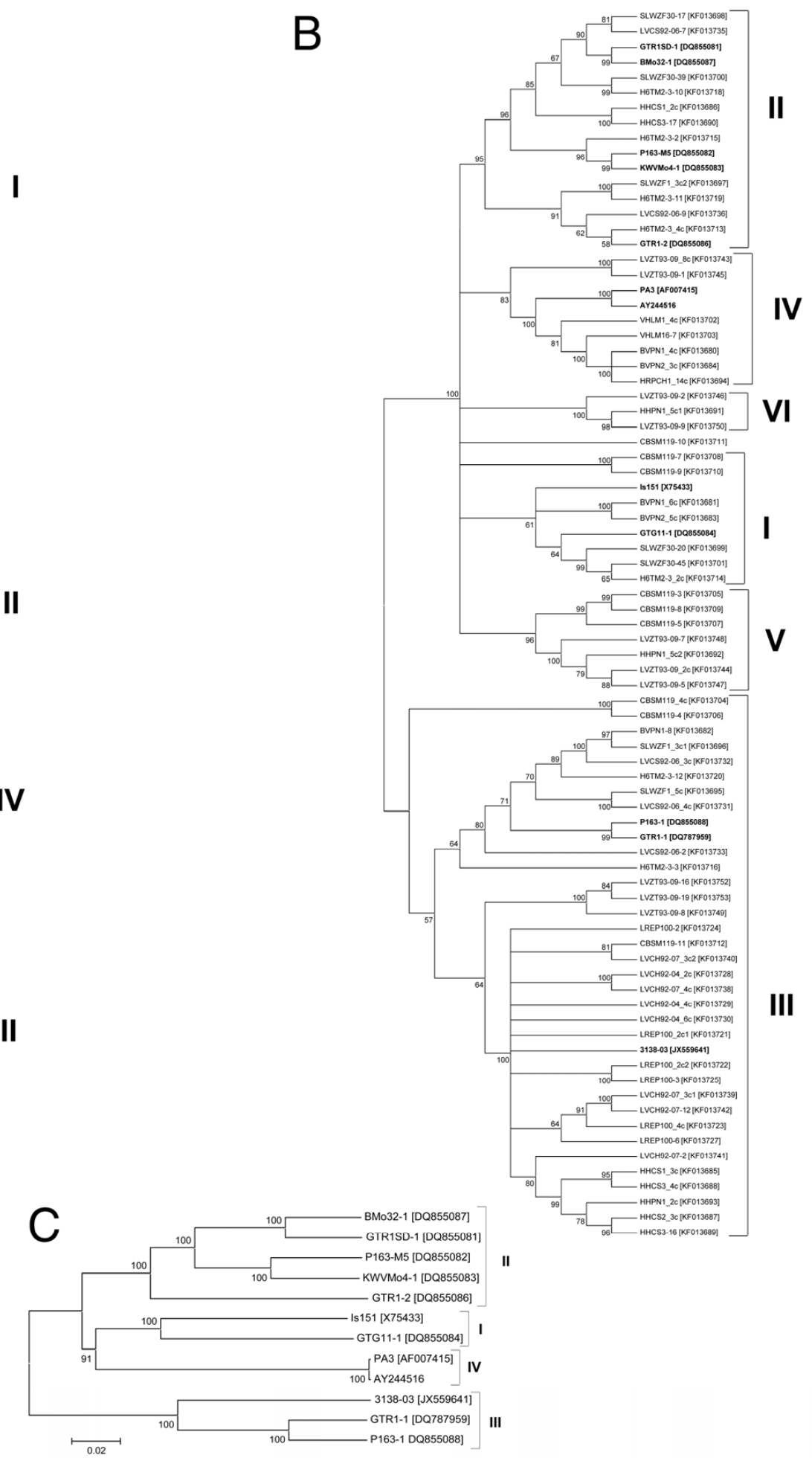

Fig. 3. Unrooted phylogenetic analysis of global isolates of Grapevine virus A (GVA) based on nucleotide sequences of the A, complete coat protein (CP); B, partial RNA-dependent RNA polymerase (RdRp); and $\mathbf{C}$, complete genome sequences. Trees were constructed by the minimum evolution (ME) using models with the lowest BIC scores from the list of models evaluated using the "Find Best DNA/Protein Models" option of MEGA5 software (52). Bootstrap values (1,000 replicates) are given at the branch nodes. Branches corresponding to partitions reproduced in $<70 \%$ (for CP), <57\% (for RdRp), and <90\% (for complete genomes) of bootstrap replicates are collapsed. Roman numerals I to VI represent individual phylogroups. The phylogroups I to IV were designated based on the relative placements of previously analyzed sequences of GVA (Table 1), while V and VI represent new phylogroups based on the RdRp data set. Sequences obtained in this study are in regular fonts while those from GenBank are in bold fonts. Details of isolates are provided in Table 1 and GenBank accession numbers of sequence haplotypes derived from these isolates are provided in square brackets in front of the sequence identifiers. 
individual grapevines ranged from two to nine and the level of nucleotide sequence identity among distinct haplotypes ranged between 72.5 and $97.6 \%$ (Table 5). For the CP, the number of distinct haplotypes present in a single grapevine ranged from two to eight and the level of nucleotide sequence identity among distinct haplotypes ranged from 74.5 to $98.44 \%$ (Table 5). Furthermore, evidence for the presence of divergent sequence variants in individual grapevines was obtained based on the distribution of distinct sequence variants from several individual grapevines in different phylogroups. For instance, analyses of the RdRp sequences from 16 isolates revealed that six isolates (LREP100, LVCH92-04, LVCH92-07, HRPCH1, HHCS2, and VHLM1) contained distinct sequence haplotypes belonging to only one phylogroup, four isolates (LVCS92-06, HHCS1, HHCS3, and BVPN2) contained haplotypes belonging to two phylogroups, five isolates (CBSM119, H6TM2-3, HHPN1, BVPN1, and SLWZF1) contained haplotypes belonging to three phylogroups, and one isolate (LVZT93-09) contained sequence haplotypes belonging to four phylogroups (Table 5). Similarly, analyses of the $\mathrm{CP}$ sequences from 26 isolates revealed that seven isolates
(LREP100, LVCH92-04, LVCH92-07, LVSB91-02, LVZT93-09, HRPCH1, and VHLM1) contained haplotypes belonging to only one phylogroup, 12 isolates (CBPR116, CBPR119, H6TM2-3, LV94-02, LVCH92-09, LVCH94-04, LVMB92-10, HHCS1, HHCS2, CSOT2, HHPN1, and BVPN1) contained haplotypes belonging to two phylogroups, and seven isolates (LVCS92-06, PACF94-142, PACF95-413, HHCS3, CSOT1, BVPN2, and SLWZF1) contained sequence haplotypes belonging to three phylogroups (Table 5). None of the isolates contained sequence haplotypes belonging to all four phylogroups.

Population genetic analysis of GVA isolates. Lower $F_{S T}$ values were obtained for both the RdRp and CP data sets when sequences of populations from California and Washington were compared with each other (Table 6). Since a value of $\mathrm{F}_{\mathrm{ST}}>0.05$ is indicative of a degree of differentiation among populations (58), our analysis suggested that gene-specific populations sampled from both California and Washington are not substantially genetically differentiated from each other. In contrast, higher $\mathrm{F}_{\mathrm{ST}}$ values between gene-specific data sets from either California or Washington and corresponding GVA sequences from other

TABLE 3. Estimates of evolutionary divergence (d) over sequence pairs of Grapevine virus A within each of the phylogroups identified in Figure 3 and summary of test statistics examined for demographic trends ${ }^{\mathrm{a}}$

\begin{tabular}{|c|c|c|c|c|c|c|c|c|c|c|c|c|}
\hline \multirow[b]{3}{*}{ Comparison } & \multicolumn{12}{|c|}{ Genetic distance and test of neutrality within groups } \\
\hline & \multicolumn{2}{|c|}{ Group I } & \multicolumn{2}{|c|}{ Group II } & \multicolumn{2}{|c|}{ Group III } & \multicolumn{2}{|c|}{ Group IV } & \multicolumn{2}{|c|}{ Group V } & \multicolumn{2}{|c|}{ Group VI } \\
\hline & $\mathrm{d}(n)$ & Tajima's D & $\mathrm{d}(n)$ & Tajima's D & $\mathrm{d}(n)$ & Tajima's D & $\mathrm{d}(n)$ & Tajima's D & $\mathrm{d}(n)$ & Tajima's D & $\mathrm{d}(n)$ & Tajima's D \\
\hline $\mathrm{RdRp}$ & $\begin{array}{l}0.155 \pm \\
0.007(9)\end{array}$ & $0.0195^{\mathrm{ns}}$ & $\begin{array}{l}0.162 \pm \\
0.006(16)\end{array}$ & $-0.0019^{\mathrm{ns}}$ & $\begin{array}{l}0.128 \pm \\
0.006(35)\end{array}$ & $-0.1967^{\mathrm{ns}}$ & $\begin{array}{l}0.121 \pm \\
0.006(9)\end{array}$ & $-0.0071^{\mathrm{ns}}$ & $\begin{array}{l}0.154 \pm \\
0.007 \text { (7) }\end{array}$ & $-0.3395^{\mathrm{ns}}$ & $\begin{array}{l}0.167 \pm \\
0.008(3)\end{array}$ & ND \\
\hline $\mathrm{CP}$ & $\begin{array}{l}0.102 \pm \\
0.008(35)\end{array}$ & $-0.2333^{\mathrm{ns}}$ & $\begin{array}{l}0.089 \pm \\
0.007(20)\end{array}$ & $0.0473^{\mathrm{ns}}$ & $\begin{array}{l}0.083 \pm \\
0.007(38)\end{array}$ & $-0.0990^{\mathrm{ns}}$ & $\begin{array}{l}0.060 \pm \\
0.006(11)\end{array}$ & $0.3957^{\mathrm{ns}}$ & NA & NA & NA & NA \\
\hline
\end{tabular}

${ }^{a}$ Results are based on the pairwise analysis of partial RNA-dependent RNA polymerase (RdRp) and complete coat protein (CP) gene sequences (GenBank accessions KF013680-754 and KF013755-854 for RdRp and CP sequences, respectively). Standard error estimates were obtained by a bootstrap procedure (1,000 replicates). Analyses were conducted in MEGA5 (52) using p-distance, and codon positions included were first, second, third, and noncoding. All positions containing gaps and missing data were eliminated from the data set (complete deletion option). Abbreviations: $n=$ number of sequences, ND $=$ not determined due to unresolved phylogeny of the gene-specific sequence data set, NA = not applicable due to absence of the phylogroup in the gene-specific sequence data set. Tajima's D statistic measures the departure from neutrality for all mutations in a genomic region (51). Values for neutrality tests were not significant (ns) in all cases $(P>0.10)$. Tajima's D statistic was performed using the DnaSP software version 5.10.01 (35).

${ }^{\mathrm{b}}$ Complete genome.

TABLE 4. Estimates of evolutionary divergence (d) over sequence pairs of Grapevine virus A (GVA) within each of the phylogroups identified in Figure 3 and summary of test statistics examined for demographic trends ${ }^{\mathrm{a}}$

\begin{tabular}{|c|c|c|c|c|}
\hline \multirow[b]{3}{*}{ Comparison } & \multicolumn{4}{|c|}{ Genetic distance between groups and test of natural selection } \\
\hline & \multicolumn{2}{|c|}{ RdRp } & \multicolumn{2}{|c|}{$\mathrm{CP}$} \\
\hline & $\mathrm{d}$ & dMKT: G ( $P$ value $)$ & $\mathrm{d}$ & dMKT: $\mathrm{G}(P$ value $)$ \\
\hline Group I vs. group III & $0.235 \pm 0.010$ & $49.50(0.00000 * * *)$ & $0.228 \pm 0.015$ & $28.90(0.000 * * *)$ \\
\hline Group I vs. group IV & $0.203 \pm 0.009$ & $1.37\left(0.24228^{\mathrm{ns}}\right)$ & $0.136 \pm 0.011$ & $0.85\left(0.35595^{\mathrm{ns}}\right)$ \\
\hline Group I vs. group V & $0.216 \pm 0.009$ & $12.82(0.00034 * * *)$ & NA & NA \\
\hline Group I vs. group VI & $0.203 \pm 0.009$ & $5.41(0.02002 *)$ & NA & NA \\
\hline Group II vs. group V & $0.228 \pm 0.009$ & $13.26(0.00027 * * *)$ & NA & NA \\
\hline Group II vs. group VI & $0.206 \pm 0.009$ & $6.03(0.01406 *)$ & NA & NA \\
\hline Group III vs. group IV & $0.247 \pm 0.011$ & 44.74 (0.00000***) & $0.222 \pm 0.014$ & 42.17 (0.000***) \\
\hline Group III vs. group V & $0.245 \pm 0.010$ & $48.68\left(0.00000^{* * *}\right)$ & NA & NA \\
\hline Group III vs. group VI & $0.252 \pm 0.010$ & $45.13\left(0.00000^{* * *}\right)$ & NA & NA \\
\hline Group IV vs. group V & $0.219 \pm 0.010$ & $9.90\left(0.00165^{* *}\right)$ & NA & NA \\
\hline Group IV vs. group VI & $0.211 \pm 0.009$ & $6.34(0.01180 *)$ & NA & NA \\
\hline
\end{tabular}

a Results are based on the pairwise analysis of partial RNA-dependent RNA polymerase (RdRp) and complete coat protein (CP) gene sequences (GenBank accessions KF013680-754 and KF013755-854 for RdRp and CP sequences, respectively). Standard error estimates were obtained by a bootstrap procedure (1,000 replicates). Analyses were conducted in MEGA5 (52) using p-distance, and codon positions included were first, second, third, and noncoding. All positions containing gaps and missing data were eliminated from the data set (complete deletion option). NA $=$ not applicable due to absence of the phylogroup in the gene-specific sequence data set. The G-test (G) statistic of the McDonald-Kreitman test (MKT) was used to determine whether synonymous and nonsynonymous variations support the hypothesis of adaptive protein evolution between two distinct variant groups (37) to determine for evidence that divergence in GVA lineages is driven by natural selection. Asterisks: * indicates $0.01<P<0.05$, ** indicates $0.001<P<0.01$, and $* * *$ indicates $P<0.001$. ns indicates not significant. MKT was performed using the DnaSP software version 5.10.01 (35). 
geographical regions (Table 6) is indicative of a substantial degree of genetic differentiation among GVA populations from North America and populations from Europe, Africa, and the Middle East. The only exception is populations from Washington and South Africa, regardless of the gene being evaluated. In this case, lower values of $\mathrm{F}_{\mathrm{ST}}$ obtained for each gene-specific comparison (Table 6) suggest that some level of genetic exchange has occurred between GVA isolates from both geographic locations probably due to exchange of vegetative cuttings. Simultaneously, we performed Hudson's Snn tests statistics which is considered more powerful than previously proposed statistics (26) and used the same thresholds described earlier for this test statistic (1). Unlike the Wrights test of genetic differentiation, significantly high values of Hudson's Snn tests statistics (26) were obtained for all sequence comparisons evaluated (Table 6) suggesting that there is high subpopulation differentiation and indicating that infrequent genetic exchange may have occurred between GVA populations from both California and Washington, and between either of the two states and other geographical regions of the world (Table 6). Based on these results, it is reasonable to conclude that infrequent genetic exchange has occurred over time between GVA populations from all geographical locations analyzed in this study.

We also evaluated nucleotide polymorphisms in each phylogroup (Fig. 3A to C) using Tajima's D statistical test (51) to determine the influence of demographic forces (55) on each genespecific data set and on complete virus genomes. Negative Tajima's D statistic indicated an excess of low-frequency polymorphism caused either by background selection, genetic hitchhiking, or

TABLE 5. Number and diversity of gene-specific haplotypes present in individual isolates analyzed in this study

\begin{tabular}{|c|c|c|c|c|c|c|c|c|c|}
\hline \multirow[b]{2}{*}{ Taxon ID } & \multirow[b]{2}{*}{ Source $^{b}$} & \multicolumn{4}{|c|}{$\operatorname{RdRp}$} & \multicolumn{4}{|c|}{$\mathrm{CP}$} \\
\hline & & $\begin{array}{l}\text { Clones } \\
(n)\end{array}$ & $\begin{array}{c}\text { Haplotypes } \\
(n)\end{array}$ & $\begin{array}{c}\text { HD } \\
(\% \mathrm{nt})\end{array}$ & $\begin{array}{l}\text { Phylogroups } \\
\qquad(n)^{\mathrm{c}}\end{array}$ & $\begin{array}{c}\text { Clones } \\
(n)\end{array}$ & $\begin{array}{c}\text { Haplotypes } \\
\text { (n) }\end{array}$ & $\begin{array}{l}\text { HD } \\
(\% \mathrm{nt})\end{array}$ & $\begin{array}{l}\text { Phylogroups } \\
(n)^{\mathrm{c}}\end{array}$ \\
\hline CBPR116 & $\mathrm{CA}$ & NA & NA & NA & NA & 6 & 4 & $75.3-90.7$ & I (3), III (1) \\
\hline CBSM119 & $\mathrm{CA}$ & 12 & 9 & $72.6-92.1$ & I (2), III (3), V (3), unknown (1) & 7 & 4 & $76.9-87.9$ & I (1), III (1), R (2) \\
\hline H6TM2-3 & $\mathrm{CA}$ & 12 & 8 & $73.6-96.5$ & I (1), II (4), III (2), R (1) & 12 & 7 & $77.3-97.1$ & I (1), II (1), R (5) \\
\hline LREP100 & $\mathrm{CA}$ & 12 & 7 & $91.4-97.4$ & III (6), R (1) & 10 & 2 & 96.9 & III (2) \\
\hline LV94-02 & $\mathrm{CA}$ & NA & NA & NA & NA & 5 & 4 & $75.3-93.6$ & I (3), III (1) \\
\hline LVCH92-04 & $\mathrm{CA}$ & 12 & 3 & $92.8-93.8$ & III (3) & 12 & 3 & $97.3-98.4$ & III (3) \\
\hline LVCH92-07 & $\mathrm{CA}$ & 12 & 5 & $90.9-97.6$ & III (5) & 9 & 5 & $90.1-98.1$ & III (5) \\
\hline LVCH92-09 & $\mathrm{CA}$ & NA & NA & NA & NA & 8 & 4 & $75.7-96.4$ & II (1), III (2), R (1) \\
\hline LVCH94-04 & $\mathrm{CA}$ & NA & NA & NA & NA & 11 & 2 & 78.8 & III (1), IV (1) \\
\hline LVCS92-06 & $\mathrm{CA}$ & 12 & 7 & $72.8-95.0$ & II (2), III (3), R (2) & 10 & 6 & $75.5-96.8$ & I (1), II (1), III (3), R (1) \\
\hline LVMB92-10 & $\mathrm{CA}$ & NA & NA & NA & NA & 10 & 5 & $74.8-97.4$ & I (3), III (1), R (1) \\
\hline LVSB91-02 & $\mathrm{CA}$ & NA & NA & NA & NA & 11 & 1 & ND & IV (1) \\
\hline LVZT93-09 & $\mathrm{CA}$ & 20 & 12 & $73.3-95.2$ & III (3), IV (2), V (3), VI (2), R (2) & 12 & 1 & ND & II (1) \\
\hline PACF94-142 & $\mathrm{CA}$ & NA & NA & NA & NA & 12 & 7 & $74.5-96.1$ & I (4), III (2), IV (1) \\
\hline PLCF95-413 & $\mathrm{CA}$ & NA & NA & NA & NA & 8 & 5 & $76.0-91.8$ & I (1), II (2), III (2) \\
\hline HRPCH1 & WA & 14 & 1 & ND & IV (1) & 10 & 1 & ND & IV (1) \\
\hline HHCS1 & WA & 5 & 2 & 76.2 & II (1), III (1) & 21 & 2 & 77.3 & II (1), III (1) \\
\hline HHCS2 & WA & 3 & 1 & ND & III (1) & 19 & 2 & 77.5 & II (1), III (1) \\
\hline HHCS3 & WA & 6 & 3 & $74.5-97.3$ & II (1), III (2) & 17 & 3 & $77.2-86.7$ & II (1), III (1), IV (1) \\
\hline CSOT1 & WA & NA & NA & NA & NA & 21 & 4 & $77.88-85.7$ & I (1), II (1), III (1), R (1) \\
\hline CSOT2 & WA & NA & NA & NA & NA & 23 & 4 & $76.8-95.9$ & I (1), III (2), R (1) \\
\hline HHPN1 & WA & 12 & 3 & $72.5-77.8$ & III (1), V (1), VI (1) & 28 & 4 & $77.2-96.1$ & I (2), III (1), R (1) \\
\hline BVPN1 & WA & 11 & 3 & $76.6-78.8$ & I (1), III (1), IV (1) & 24 & 3 & $76.8-90.7$ & I (2), III (1) \\
\hline BVPN2 & WA & 8 & 2 & 78.5 & I (1), IV (1) & 24 & 5 & $74.7-89.7$ & I (2), II (1), III (1), R (1) \\
\hline VHLM1 & WA & 5 & 2 & 90 & IV (2) & 9 & 4 & 95.9-97.9 & IV (4) \\
\hline SLWZF1 & WA & 15 & 7 & $73.7-88.88$ & I (2), II (3), III (2) & 24 & 8 & $75.7-96.3$ & I (2), II (3), III (2), R (1) \\
\hline
\end{tabular}

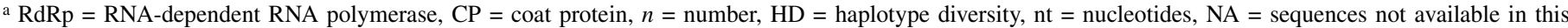
genomic region for this isolate, and ND = not determined because all sequences represent a single haplotype.

b $\mathrm{CA}=$ California and $\mathrm{WA}=$ Washington.

${ }^{c}$ Number of sequences in parentheses. $\mathrm{R}=$ recombinant sequences.

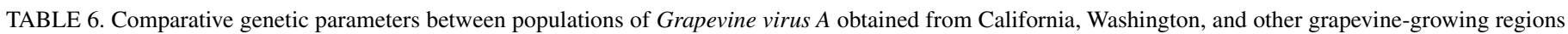
of the world ${ }^{\mathrm{a}}$

\begin{tabular}{|c|c|c|c|}
\hline Gene, comparison $^{\mathrm{b}}$ & $\mathrm{F}_{\mathrm{ST}}$ & Snn & $P$ value \\
\hline \multicolumn{4}{|l|}{$\operatorname{RdRp}$} \\
\hline California vs. Washington & 0.02687 & 0.68841 & $0.0310 *$ \\
\hline California vs. South Africa & 0.07832 & 0.81132 & $0.1220^{\mathrm{ns}}$ \\
\hline California vs. Israel & 0.55931 & 1.00000 & $0.0050 * *$ \\
\hline Washington vs. South Africa & 0.04121 & 0.83750 & $0.0060 * *$ \\
\hline Washington vs. Israel & 0.49846 & 0.96154 & $0.02300 *$ \\
\hline \multicolumn{4}{|l|}{$\mathrm{CP}$} \\
\hline California vs. Washington & 0.02326 & 0.87451 & $0.0000 * * *$ \\
\hline California vs. South Africa & 0.08032 & 0.87432 & $0.001000 * *$ \\
\hline California vs. Israel & 0.47305 & 1.00000 & $0.0100000^{*}$ \\
\hline California vs. Czech Republic & 0.27246 & 0.96154 & $0.099000^{\mathrm{ns}}$ \\
\hline Washington vs. South Africa & 0.02733 & 0.91304 & $0.0000 * * *$ \\
\hline Washington vs. Israel & 0.43404 & 1.00000 & $0.0110000^{*}$ \\
\hline Washington vs. Czech Republic & 0.25056 & 0.94595 & $0.070000^{\mathrm{ns}}$ \\
\hline
\end{tabular}

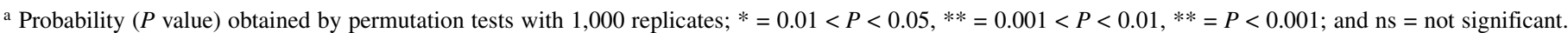
All analyses were performed using the DnaSP software version 5.10.01 (35).

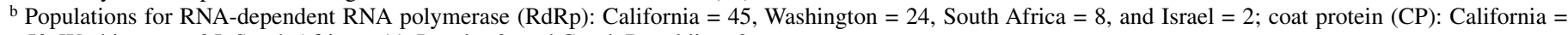
50 , Washington $=35$, South Africa $=11$, Israel $=2$, and Czech Republic $=2$. 
population expansions $(1,55)$. In contrast, positive values of Tajima's D statistic suggested low levels of low and high frequency polymorphisms, indicating a decrease in population size and/or balancing selection. The analyses yielded negative values of Tajima's D statistic for groups II, III, IV, and V for the RdRp data set, groups I and III for the CP data set, and group II for the complete genome data set. In contrast, positive Tajima's D statistic was obtained for group I of RdRp and groups II and IV of $\mathrm{CP}$ (Table 3). However, it should be noted that these values are not statistically significant $(P>0.10)$ in all cases; hence it is plausible to hypothesize that purifying selection may be acting on each of the GVA variant populations. We could not perform Tajima's D statistic test for complete genome sequences of GVA in group I, III and IV, since at least four sequences are needed for this analysis in the DnaSP software. Similarly, the lack of phylogroups V and VI in the $\mathrm{CP}$ and complete genome data sets and presence of only three sequences in the RdRp-specific phylogroup VI made it difficult to evaluate Tajima's D statistic for these subsets of GVA sequence variants. Finally, we used the McDonald and Kreitman test (37) to test the hypothesis that the evolutionary history of gene-specific phylogroups of GVA (Fig. 3) have been shaped by natural selection acting on each protein-encoding gene. Results of the test showed significant differences (with good statistical support based on $P$ values of Fisher's and G test) for both RdRp and CP sequence data sets for most of the phylogroup comparisons (Table 4). This indicates that natural selection contributed to the evolution of variants belonging to majority of the RdRp- and CP-based phylogroups identified in this study. In contrast, we did not find significant Fisher's and G-test statistics, for both RdRp and $\mathrm{CP}$, when comparisons were made between sequence variants belonging to phylogroups I and IV and between groups II and IV for the CP data set alone (Table 4). These results suggest that the divergence of sequences belonging to phylogroup IV from group I in both genomic regions, and phylogroup IV from group II in the $\mathrm{CP}$ alone, may be a consequence of random processes.

Selection constraints on RdRp and CP. The types of evolutionary forces acting on $\mathrm{RdRp}$ and $\mathrm{CP}$ sequences of GVA were determined by computing the normalized $\mathrm{d} N-\mathrm{d} S$ values for each genomic region (Table 7). The results showed that the normalized $\mathrm{d} N-\mathrm{d} S$ values for the RdRp and CP genes were 0.0264 and 0.0505 , respectively. Further analysis revealed a preponderance of codon sites undergoing negative selection in both the RdRp $(235 / 250$ or $\approx 94 \%)$ and CP $(155 / 198$ or $\approx 78 \%)$, and no evidence for any positively selected sites in both genes (Table 7). Based on these results, it appears that both coding regions are subjected to the influence of very strong purifying selection.

\section{DISCUSSION}

In this study, we have determined the genetic diversity of field isolates of GVA derived from California and Washington vineyards using partial replicase (RdRp) and complete CP sequences.
The data indicated that GVA is present as genetically distinct variants in different cultivars from the two major grapevinegrowing regions in the United States. Further, our results confirmed previous reports from grapevine-growing regions outside the United States on the occurrence of mixed infections of highly divergent variants of GVA in individual grapevines $(19,20,21$, $43,57)$. Since genetic diversity within natural populations of GVA from American vineyards have received less attention in previous studies, the results of this study have advanced our knowledge of genetic diversity of GVA on a global scale for a better understanding of the epidemiology of the virus as a prerequisite to implementing appropriate management strategies to minimize spread of genetically distinct strains of the virus by vectors and via planting materials. From a practical point of view, the genetic diversity data derived from this study showed that CP-specific primers GVA-CPF6356 and GVA-CPR7096 were more robust and able to detect all isolates of GVA used in this study than primers GVA944F (5'-GACAAATGGCACACTACG) and GVA1353R (5'-AAGCCTGACCTAGTCATCTTGG) (40) currently being used for routine diagnosis of the virus. Thus, the GVA-CPF6356 and GVA-CPR7096 primer pair will enable an effective monitoring of GVA in grower vineyards and grape clean plant programs.

Recent studies by Wang et al. (57), based on the overall nucleotide identity of the CP, and Goszczynski and Habili (20), based on a $293 \mathrm{nt}$ variable part of the replicase gene, reported clustering of GVA isolates into three phylogenetic groups. In contrast, our results, based on complete CP gene sequences, revealed segregation of natural populations of GVA into four distinct phylogenetic groups, with isolates from California and Washington dispersed in all four groups irrespective of their host cultivar or origin (Fig. 3A). When RdRp-specific sequences were used for phylogenetic analysis, GVA isolates segregated into six phylogroups (Fig. 3B). Such an incongruent phylogeny of GVA isolates would suggest considerable sequence heterogeneity in the $\mathrm{RdRp}$ relative to the $\mathrm{CP}$ gene sequences. These observations would indicate the likely occurrence of intergenic recombinations between the genome of distinct variants present as mixed infections in a single grapevine. Since the RdRp and CP sequences analyzed in this study represent only about $20 \%$ of the GVA genome, further studies are required to identify recombination hotspots along the virus genome for quantitative estimates of the frequency and kinetics of appearance of recombinants in natural populations of GVA.

The presence of genetically diverse sequence variants of GVA in individual grapevines (Table 5) is in agreement with previous studies $(19,20,43,57)$. This conclusion is supported by considerable haplotype diversities within individual grapevines and segregation of gene-specific sequence variants obtained from individual grapevines into divergent phylogroups (Table 5; Fig. 3A and B). The existence of sequence variant mixtures during natural infections have also been documented for other grapevine viruses

TABLE 7. Estimates of selection pressures acting on the RNA-dependent RNA polymerase (RdRp) and coat protein (CP) genes of global isolates of Grapevine virus A (GVA)

\begin{tabular}{|c|c|c|c|c|c|c|c|c|}
\hline \multirow[b]{3}{*}{ Coding region ${ }^{\mathrm{a}}$} & \multicolumn{8}{|c|}{ Selection pressure ${ }^{b}$} \\
\hline & \multicolumn{2}{|c|}{ Normalized $\mathrm{d} N-\mathrm{d} S^{\mathrm{c}}$} & \multicolumn{2}{|c|}{ Positive } & \multicolumn{2}{|c|}{ Negative } & \multicolumn{2}{|c|}{ Neutral } \\
\hline & $\log (\mathrm{L})$ & Mean & Number & Percent & Number & Percent & Number & Percent \\
\hline $\mathrm{CP}$ & $-8,609.12$ & 0.0505 & 0 & 0 & 155 & 78.3 & 43 & 21.7 \\
\hline
\end{tabular}


including Grapevine rupestris stem pitting-associated virus (2), Grapevine fanleaf virus (38), and several grapevine leafrollassociated viruses $(1,9,29,56)$. As discussed in a previous study (2), mixed infections could be the consequence of grafting in which the scion and rootstock harboring distinct variants were brought together into individual grafted plants. Additionally, inoculation by mealybugs and scale insects could also be contributing to co-infections of GVA variants, when these vectors are able to introduce divergent sequence variants from one plant to another during transmission within and between vineyards. Clonal propagation, the perennial nature of grapevines and the error-prone nature of the viral RdRp (6), which leads to a high error frequency for replication of RNA viruses varying between $10^{-3}$ and $10^{-5}$ misincorporations per nucleotide copied (8), would promote evolution of genetic variants within an individual plant, thereby increasing intra-plant population diversity. In addition, a complex interplay between diverse aspects of virus biology, such as genomic architecture, mode and speed of replication and the rate at which genomes generate and fix mutations (8) could also contribute to the overall within-host population diversity of GVA. Thus, GVA, like other grapevine viruses, can perpetuate as genetically diverse populations known as a quasispecies (5). It should be noted, however, that populations of GVA can be subjected to repeated bottleneck events during their life cycle, such as cell-tocell movement and systemic spread within the host plant and during horizontal vector-mediated transmissions $(3,12,34,49)$. As a woody perennial adapted to temperate-zone climate, grapevine undergoes alternating periods of active growth from spring to fall and dormancy during winter. Therefore, unlike annual plant species, population dynamics of viruses in grapevine may be subjected to seasonal changes and environmental vagaries during the cropping season in a given physical environment (28). Consequently, the overall genetic structure of GVA populations within an individual grapevine is dynamic and may be shaped by hostand vector-imposed bottlenecks as well as the extent of a possible "compartmentalization" of progeny virus within the plant during each growing season (30). Growing evidence also indicates that mixed virus infections and superinfection exclusion in infected cells could impose additional bottlenecks in the tissue tropism and perpetuation of virus diversity in perennial plants (10). Although many of these mutant virus populations are "purged" from the source grapevine while pruning soon after dormancy, new variants can be generated anew during each growing season. Additionally, most of these genetic variants can be disseminated annually from mother to progeny plants via cuttings used for new plantings and new generations of variants can be produced within each individual descendent.

The non-tree-like phylogenetic networks among RdRp- and CP-specific sequences of GVA (Fig. 2) implicate recombination as an important process in the genetic diversity of GVA. A test of this hypothesis led to the detection of putative recombination events and the identification of potential recombinants and their parental sequences among GVA sequence variants (Table 2). It is unlikely that these recombination events arose due to sequencing errors, since multiple independent clones originating from a specific RT-PCR product were used for sequence analyses. The identification of recombinant breakpoints at similar positions in different sequence variants by at least four of the seven recombination detection programs in the RDP3, coupled with highly significant statistical support obtained for each event (Table 2), indicate that sequencing or assembling errors should be of minimal effect in the inferred recombination events. What is significant from our study is the evidence that majority of putative recombination events identified in the $\operatorname{RdRp}$ sequences were mostly derived from intra-isolate recombinations in contrast to inter-isolate parentals contributing to these events in the CP. The underlying mechanistic factors in inferred differences in RdRp and $\mathrm{CP}$ sequences remain unclear and warrant further studies. It could be that distinct functional roles of RdRP and $\mathrm{CP}$ and their discrete location in the virus genome, the selective constraints experienced in the two genomic regions during the virus replication cycle and genomic secondary structures are contributing to the genetic fitness of inter-isolate recombination events occurring in the CP than in the RdRp. Presumably, subgenomic RNAs expressing the $\mathrm{CP}$ generated during virus replication (13) provide additional advantages for increased fitness of recombination events in the CP. As indicated earlier, it is plausible that besides the $\mathrm{RdRp}$ and $\mathrm{CP}$, recombinogenic regions might be present in other genomic regions of GVA and additional sequencing on whole-genome scale will be required to identify discrete recombination hot-spots across the virus genome and comprehend the extent and role of recombination in genetic diversity and evolution of GVA.

An investigation of functional constraints on the evolution of RdRp and CP genes of GVA was made by calculating the normalized $\mathrm{d} N-\mathrm{d} S$ values which is considered an indicator of natural selection. This analysis revealed a lack of positive selection acting on both gene sequences and indicated that these genes are under strong selection pressure to preserve their integrity and biological functions. Interestingly, the $\mathrm{RdRp}$ gene had the lowest mean normalized $\mathrm{d} N-\mathrm{d} S$ value with 94 and $6 \%$ of its 250 codon sites undergoing negative and neutral selection, respectively (Table 7). This suggests that the partial RdRp gene fragment analyzed in this study is under strong purifying selection. On the other hand, only 78.3 and $21.7 \%$ of the $198 \mathrm{CP}$ codon sites were negatively and neutrally selected, respectively (Table 7). Thus, strong signal of purifying selection for the majority of codons in both genes indicate purifying selection as a dominating feature in shaping the evolution of GVA. Although lower than values obtained for RdRp coding sequences, the percentage of $\mathrm{CP}$-specific negatively selected sites of GVA is considerably greater than values obtained for other grapevine viruses transmitted by the same vector species, such as GLRaV-1 (1) and GLRaV-3 (56).

Phylogenetic separation of GVA isolates into four lineages based on the CP sequences (Fig. 3A), in contrast to the topology of the RdRp tree (Fig. 3B), supported the overall topology of the phylogenetic tree derived from complete genome sequences of the virus (Fig. 3C). This suggests that the $\mathrm{CP}$ gene sequences are more amenable for genetically based classification of GVA isolates into four lineages than $\mathrm{RdRp}$ sequences. The relative ease of amplification of the complete $\mathrm{CP}$ coding sequences from different GVA isolates (Fig. 1B) will be particularly helpful in future studies for type assignment of new virus isolates based on the $\mathrm{CP}$ sequences without recourse to generating the complete genome sequences. Conversely, analyzing sequences from discrete regions of the GVA genome, including the $\mathrm{CP}$ and RdRp, would be valuable in understanding the phylogenetic history of virus populations. Similarly, information on recombination patterns in different portions of the GVA genome will assist the interpretation of sequence data for a better understanding of genetic diversity and evolutionary history of the virus. Although the CPand RdRp-based phylogeny of GVA (Fig. 3A and B) excluded putative recombinant sequences, it is unlikely that elimination of these small numbers of sequences (Table 2, Fig. 2A and B) negatively influenced the robustness of phylogenetic analysis presented in this study. Nevertheless, identification of additional recombinant sequences in future studies will help to rigorously analyze phylogenetic history of GVA by including all component parts of the virus-both normal and putative recombinant sequences.

Previous studies have linked group II sequence variants of GVA with SD in both own-rooted and grafted grapevines grown in South African (18) and Australian (20) vineyards. Although several sequence variants belonging to phylogroup II are present in Washington and California vineyards based on their clustering relative to global isolates of GVA (Fig. 3), we have not found 
evidence for the presence of SD in both locations. A plausible explanation for this is that GVA sequences analyzed in this study were derived from grapevines mixed infected with other viruses (data not shown) and such mixed infections could be masking the expression of SD symptoms.

In conclusion, this study represents the most comprehensive analysis to date of the genetic diversity and population genetics of GVA isolates from two major grape-producing regions within the United States. The information from this study will provide a baseline for applied research to advance our understanding of the epidemiology of GVA in grower vineyards and implement appropriate management strategies for minimizing the spread of genetic variants of the virus by vectors and via planting materials supplied to nurseries and grape growers. In addition, the extensive sequence data generated in this study will enable the design of better diagnostic assays for use in grape clean plant programs to ensure reliable supply and propagation of clean plant materials.

\section{ACKNOWLEDGMENTS}

This work was supported, in part, by Washington State University's Agricultural Research Center in the College of Agricultural, Human, and Natural Resource Sciences, Members of the Wine Advisory Committee of the Washington Wine Commission, Northwest Center for Small Fruits Research and Viticulture Consortium-West. PPNS number 0623, Department of Plant Pathology, College of Agricultural, Human, and Natural Resource Sciences, Agricultural Research Center Project Number WNPO 0616, Washington State University, Pullman 99164-6240. We thank A. Rowhani (Department of Plant Pathology, University of California, Davis) for encouragement and critical review of the manuscript, R. C. Larsen (Washington State University, Irrigated Agriculture Research and Extension Center, Prosser) for critical review of the manuscript and anonymous reviewers for helpful suggestions.

\section{LITERATURE CITED}

1. Alabi, O. J., Al Rwahnih, M., Karthikeyan, G., Poojari, S., Fuchs, M., Rowhani, A., and Naidu, R. A. 2011. Grapevine leafroll-associated virus 1 occurs as genetically diverse populations. Phytopathology 101:1446-1456.

2. Alabi, O. J., Martin, R. R., and Naidu, R. A. 2010. Sequence diversity, population genetics and potential recombination events in Grapevine rupestris stem pitting-associated virus in Pacific North-West vineyards. J. Gen. Virol. 91:265-276.

3. Ali, A., and Roossinck, M. J. 2008. Genetic bottlenecks. Pages 123-131 in: Plant Virus Evolution. M. J. Roossinck, ed. Springer-Verlag, Berlin, Heidelberg, Germany.

4. Conti, M., Milne, R. G., Luisoni, E., and Boccardo, G. 1980. A closterovirus from a stem-pitting diseased grapevine. Phytopathology 70:394-399.

5. Domingo, E. 2002. Quasispecies theory in virology. J. Virol. 76:463-465.

6. Drake, J. W., and Holland, J. J. 1999. Mutation rates among RNA viruses. Proc. Natl. Acad. Sci. USA 96:13910-13913.

7. Du Preez, J., Stephan, D., Mawassi, M., and Burger, J. T. 2011. The grapevine-infecting vitiviruses, with particular reference to Grapevine virus A. Arch. Virol. 156:1495-1503.

8. Duffy, S., Shackelton, L. A., and Holmes, E. C. 2008. Rates of evolutionary change in viruses: Patterns and determinants. Nat. Rev. Gen. 9:267-276.

9. Farooq, A. B. U., Maa, Y., Wanga, Z., Zhuoa, N., Wenxing, X., Wanga, G., and Hong, N. 2013. Genetic diversity analyses reveal novel recombination events in Grapevine leafroll-associated virus 3 in China. Virus Res. 171:15-21.

10. Folimonova, S. Y., Robertson, C. J., Shilts, T., Folimonov, A. S., Hilf, M. E., Garnsey, S. M., and Dawson, W. O. 2010. Infection with strains of Citrus tristeza virus does not exclude superinfection by other strains of the virus. J. Virol. 84:1314-1325.

11. Fortusini, A., Scattini, G., Prati, S., Cinquanta, S., and Belli, G. 1997. Transmission of grapevine leafroll virus 1 (GLRV-1) and Grapevine virus A (GVA) by scale insects. Pages 121-122 in: Extended Abstracts, 12th Meeting of the International Council for the Study of Virus and Virus-like Diseases of the Grapevine, Lisbon, Portugal.

12. French, R., and Stenger, D. C. 2003. Evolution of Wheat streak mosaic virus: Dynamics of population growth within plant may explain limited variation. Annu. Rev. Phytopathol. 41:199-214.

13. Galiakparov, N., Goszczynski, D. E., Che, X., Batuman, O., Bar-Joseph, M., and Mawassi, M. 2003. Two classes of subgenomic RNA of Grape- vine virus A produced by internal controller elements. Virology 312:434448.

14. Galiakparov, N., Tanne, E., Mawassi, M., Gafny, R., and Sela, I. 2003. ORF 5 of Grapevine virus $A$ encodes a nucleic acid-binding protein and affects pathogenesis. Virus Genes 27:257-262.

15. Galiakparov, N., Tanne, E., Sela, I., and Gafny, R. 2003. Functional analysis of the Grapevine virus A genome. Virology 306:42-50.

16. Garau, R., Prota, V. A., Boscia, D., Fiori, M., and Prota, U. 1995. Pseudococcus anis Mask., a new vector of trichoviruses A and B. Vitis 34:67-68.

17. Golino, D. A. 1992. The Davis grapevine virus collection. Am. J. Enol. Vitic. 43:200-205

18. Goszczynski, D. E. 2007. Single-strand conformation polymorphism SSCP, cloning and sequencing of Grapevine virus A (GVA) reveal a close association between related molecular variants of the virus and Shiraz disease in South Africa. Plant Pathol. 56:755-762.

19. Goszczynski, D. E., du Preez, J., and Burger, J. T. 2008. Molecular divergence of Grapevine virus A (GVA) variants associated with Shiraz disease in South Africa. Virus Res. 138:105-110.

20. Goszczynski, D. E., and Habili, N. 2012. Grapevine virus A variants of group II associated with Shiraz disease in South Africa are present in plants affected by Australian Shiraz disease, and have also been detected in the USA. Plant Pathol. 61:205-214.

21. Goszczynski, D. E., and Jooste, A. E. C. 2003. Identification of grapevines infected with divergent variants of Grapevine virus A using variantspecific RT-PCR. J. Virol. Methods 112:157-164.

22. Goszczynski, D. E., and Jooste, A. E. C. 2003. Shiraz disease is transmitted by mealybug Planococcus ficus and associated with Grapevine virus A. Page 219 in: Extended Abstracts of the XIV Meeting of the International Council for the Study of Viruses and Virus-like Diseases of the Grapevines, Locorotondo, Italy.

23. Grasso, C., and Lee, C. 2004. Combining partial order alignment and progressive multiple sequence alignment increases alignment speed and scalability to very large alignment problems. Bioinformatics 20:15461556.

24. Haviv, S., Moskovitz, Y., and Mawassi, M. 2012. The ORF3-encoded proteins of vitiviruses GVA and GVB induce tubule-like and punctate structures during virus infection and localize to the plasmodesmata. Virus Res. 163:291-301.

25. Hommay, G., Komar, V., Lemaire, O., and Herrbach, E. 2008. Grapevine virus A transmission by larvae of Parthenolecanium corni. Eur. J. Plant Pathol. 121:185-188.

26. Hudson, R. R. 2000. A new statistic for detecting genetic differentiation. Genetics 155:2011-2014.

27. Huson, D. H., and Bryant, D. 2006. Application of phylogenetic networks in evolutionary studies. Mol. Biol. Evol. 23:254-267.

28. Jaag, H. M., and Nagy, P. D. 2010. The combined effect of environmental and host factors on the emergence of viral RNA recombinants. PLoS Pathog. 6:e1001156.

29. Jarugula, S., Alabi, O. J., Martin, R. R., and Naidu, R. A. 2010. Genetic variability of natural populations of Grapevine leafroll-associated virus 2 in Pacific Northwest vineyards. Phytopathology 100:698-707.

30. Jridi, C., Martin, J.-F., Marie-Jeanne, V., Labonne, G., and Blanc, S. 2006. Distinct viral populations differentiate and evolve independently in a single perennial host plant. J. Virol. 80:2349-2357.

31. Kosakovsky Pond, S. L., and Frost, S. D. W. 2005. Datamonkey: Rapid detection of selective pressure on individual sites of codon alignments. Bioinformatics 21:2531-2533.

32. Kosakovsky Pond, S. L., Posada, D., Gravenor, M. B., Woelk, C. H., and Frost, S. D. 2006. GARD: A genetic algorithm for recombination detection. Bioinformatics 22:3096-3098.

33. La Notte, P., Buzkan, N., Choueiri, E., Minafra, A., and Martelli, G. P. 1997. Acquisition and transmission of Grapevine virus $A$ by the mealybug Pseudococcus longispinus. J. Plant Pathol. 78:79-85.

34. Li, H. Y., and Roossinck, M. J. 2004 Genetic bottlenecks reduce population variation in an experimental RNA virus population. J. Virol. 78:10582-10587.

35. Librado, P., and Rozas, J. 2009. DnaSP v5: A software for comprehensive analysis of DNA polymorphism data. Bioinformatics 25:1451-1452.

36. Martin, D. P., Williamson, C., and Posada, D. 2005. RDP2: Recombination detection and analysis from sequence alignments. Bioinformatics $21: 260-262$.

37. McDonald, J. L., and Kreitman, M. 1991. Adaptive protein evolution at the Adh locus in Drosophila. Nature 351:652-654.

38. Mekuria, T. A., Gutha, L. R., Martin R. R., and Naidu R. A. 2009. Genome diversity and intra- and inter-species recombination events in Grapevine fanleaf virus. Phytopathology 99:1394-1402.

39. Minafra, A. 2000. Rugose wood of grapevines. Pages 30-34 in: Extended Abstracts of the 13th Meeting of the International Council for the Study of Viruses and Virus-like Diseases of the Grapevines, Adelaide, Australia.

40. Minafra, A., Saldarelli, P., Grieco, F., and Martelli, G. P. 1994. Nucleotide 
sequence of the $3^{\prime}$ terminal region of the RNA of two filamentous grapevine viruses. Arch. Virol. 137:249-261.

41. Minafra, A., Saldarelli, P., and Martelli, G. P. 1997. Grapevine virus A: Nucleotide sequence, genome organization, and relationship in the trichovirus genus. Arch. Virol. 142:417-423

42. Monette, P. L., Godkin, S. E., and James, D. 1990. Mechanical sap transmission of a closterovirus from in vitro shoot tip cultures of a leafroll-affected grapevine to Nicotiana benthamiana. Vitis 29:49-55.

43. Murolo, S., Romanazzi, G., Rowhani, A., Minafra, A., La Notte, P., Branzanti, M., and Savino, V. 2008. Genetic variability and population structure of Grapevine virus A coat protein gene from naturally infected Italian vines. Eur. J. Plant Pathol. 120:137-145.

44. Nei, M., and Kumar, S. 2000. Molecular Evolution and Phylogenetics. Oxford University Press, New York.

45. Posada, D., and Crandall, K. A. 2002. The effect of recombination on the accuracy of phylogeny estimation. J. Mol. Evol. 54:396-402.

46. Rosciglione, B., and Castellano, M. A. 1985. Further evidence that mealybugs can transmit Grapevine virus A (GVA) to herbaceous hosts. Phytopathol. Mediter. 24:186-188.

47. Rowhani, A., Biardi, L., Johnson, R., Saldarelli, P., Zhang, Y. P., Chin, J., and Green, M. 2000. Simplified sample preparation method and one-tube RT-PCR for grapevine viruses. Page 82 in: Extended Abstracts of the 13th Meeting of the International Council for the Study of Viruses and Viruslike Diseases of the Grapevines, Adelaide, Australia.

48. Rzhetsky, A., and Nei, M. 1992. A simple method for estimating and testing minimum-evolution trees. Mol. Biol. Evol. 9:945-967.

49. Sacristan, S., Malpica, J. M., Fraile, A., and Garcia-Arenal, F. 2003. Estimation of population bottlenecks during systemic movement of Tobacco mosaic virus in tobacco plants. J. Virol. 77:9906-9911.

50. Saitou, N., and Nei, M. 1987. The neighbor-joining method: A new method for reconstructing phylogenetic trees. Mol. Biol. Evol. 4:406-425.

51. Tajima, F. 1989. Statistical method for testing the neutral mutation hypothesis by DNA polymorphism. Genetics 123:585-595.

52. Tamura, K., Peterson, D., Peterson, N., Stecher, G., Nei, M., and Kumar, S. 2011. MEGA5: Molecular evolutionary genetics analysis using maximum likelihood, evolutionary distance, and maximum parsimony methods. Mol. Biol. Evol. 28:2731-2739.

53. Tanne, E., Ben-Dov, Y., and Raccah, B. 1989. Transmission of the corkybark disease by the mealybug Planococcus ficus. Phytoparasitica 17:55.

54. Thompson, J. D., Higgins, D. G., and Gibson, T. J. 1994. CLUSTAL W: Improving the sensitivity of progressive multiple sequence alignment through sequence weighting, positions-specific gap penalties and weigh matrix choice. Nucleic Acid Res. 22:4673-4680.

55. Tsompana, M., Abad, J., Purugganan, M., and Moyer, J. W. 2005. The molecular population genetics of the Tomato spotted wilt virus (TSWV) genome. Mol. Ecol. 14:53-66.

56. Wang, J., Sharma, A. M., Duffy, S., and Almeida, R. P. P. 2011.Genetic diversity in the $3^{\prime}$ terminal 4.6-kb region of Grapevine leafroll-associated virus 3. Phytopathology 101:445-450.

57. Wang, Z., Hong, N., Liu, Y., Xu, W., and Wang, G. 2011. Genetic variability and population structure of Grapevine virus $A$ in China based on the analysis of its coat protein gene. Can. J. Plant Pathol. 33:227-233.

58. Wright, S. 1951. The genetical structure of populations. Ann. Eugen. $15: 323-354$.

59. Zhou, Z., Dell'Orco, M., Saldarelli, P., Turturo, C., Minafra, A., and Martelli, G. P. 2006. Identification of an RNA-silencing suppressor in the genome of Grapevine virus A. J. Gen. Virol. 87:2387-2395.

60. Zorloni, A., Prati, S., Bianco, P. A., and Belli, G. 2006. Transmission of Grapevine virus $A$ and Grapevine leafroll-associated virus 3 by Heliococcus bohemicus. J. Plant Pathol. 88:325-328. 